\title{
Error-correcting neural networks for semi-Lagrangian advection in the level-set method
}

\author{
Luis Ángel Larios-Cárdenas ${ }^{\mathrm{a}, *}$, Frédéric Gibou ${ }^{\mathrm{a}, \mathrm{b}}$ \\ ${ }^{a}$ Computer Science Department, University of California, Santa Barbara, CA 93106, USA \\ ${ }^{b}$ Mechanical Engineering Department, University of California, Santa Barbara, CA 93106, USA
}

\begin{abstract}
We present a machine learning framework that blends image super-resolution technologies with passive, scalar transport in the level-set method. Here, we investigate whether we can compute on-the-fly, data-driven corrections to minimize numerical viscosity in the coarse-mesh evolution of an interface. The proposed system's starting point is the semi-Lagrangian formulation. And, to reduce numerical dissipation, we introduce an error-quantifying multilayer perceptron. The role of this neural network is to improve the numerically estimated surface trajectory. To do so, it processes localized level-set, velocity, and positional data in a single time frame for select vertices near the moving front. Our main contribution is thus a novel machine-learning-augmented transport algorithm that operates alongside selective redistancing and alternates with conventional advection to keep the adjusted interface trajectory smooth. Consequently, our procedure is more efficient than full-scan convolutional-based applications because it concentrates computational effort only around the free boundary. Also, we show through various tests that our strategy is effective at counteracting both numerical diffusion and mass loss. In simple advection problems, for example, our method can achieve the same precision as the baseline scheme at twice the resolution but at a fraction of the cost. Similarly, our hybrid technique can produce feasible solidification fronts for crystallization processes. On the other hand, tangential shear flows and highly deforming simulations can precipitate bias artifacts and inference deterioration. Likewise, stringent design velocity constraints can limit our solver's application to problems involving rapid interface changes. In the latter cases, we have identified several opportunities to enhance robustness without forgoing our approach's basic concept. Despite these circumstances, we believe all the above assets make our framework attractive to parallel level-set algorithms. Its appeal resides in the possibility of avoiding further mesh refinement and decreasing expensive communications between computing nodes.
\end{abstract}

Keywords: machine learning, semi-Lagrangian advection, error modeling, neural networks, level-set method, Stefan problem

\section{Introduction}

In the last few years, we have witnessed unprecedented progress in scientific disciplines thanks to machine learning. Advancements in these areas have sped up, especially with the democratization of computing resources and increased data accessibility. In particular, thriving machine learning applications [1-3] have emerged from computer vision and image processing [4-12], computer graphics [13], natural language processing and information retrieval [14-17], and sequencing and language translation [18-20]. Not long ago, machine learning and neural networks began carving their paths through the classic numerical methodologies, leading to promising ramifications in mathematics, physics, and engineering [21]. It all started with the groundbreaking work by Lagaris et al. [22, 23] to approximate the solution to boundary-value problems.

More recent machine learning incursions in computational science have brought about a broad spectrum of novel applications. Among these, physics-informed neural networks (PINNs) [24-26] have fueled a large body of scientific deep learning developments. The motivation behind PINNs is to uncover the governing dynamics in spatiotemporal data sets (e.g., $[27,28])$. Also, another group of techniques has found a niche in conservation-law simulations. Contributions in this branch include classifiers for near-discontinuity regions [29], shock detectors for complex flows [30],

${ }^{*}$ Corresponding author

Email addresses: lal@cs.ucsb.edu (Luis Ángel Larios-Cárdenas), fgibou@ucsb.edu (Frédéric Gibou) 
and under-resolved-stencil discriminators for surface reconstruction [31]. These models have assisted with balancing efficiency and accuracy by enabling their systems to switch between solvers depending on field smoothness and other attributes.

Practitioners, too, have turned to machine learning to address challenging tasks in free-boundary problems (FBPs) [32]. Some implicit formulations widely used for solving FBPs are the volume-of-fluid (VOF) [33], the phase-field $[34,35]$, and the level-set methods [36]. In VOF technologies, we can find a representative combination of computational fluid dynamics (CFD) and machine learning in the work by Després and Jourdren with algorithms for bimaterial compressible Euler calculations [37]. Similarly, Qi et al. [38] and Patel et al. [39] have optimized neural networks to estimate curvature at the center of two- and three-dimensional volume-fraction stencils. Besides curvature computation, the interface geometry is likewise hard to characterize because of the discontinuous nature of the VOF representation. Ataei et al. [40], for example, have proposed a neural piece-wise linear interface-construction (NPLIC) system to deal with this difficulty. Their alternative approach has outperformed the complex analytical PLIC procedures [41] while being nearly as accurate at faster evaluation rates.

The level-set method has also benefited from data-driven technologies. In this formulation, researchers have actively sought efficacious solutions to long-standing difficulties derived from the absence of inbuilt mechanisms that secure well-balancedness [42] and preserve mass [43]. Such problems can get exacerbated in coarse meshes and when the free boundary undergoes severe stretching or tearing [44]. First, well-balancedness relates to surface tension models and their ability to recover equilibrium solutions [42]. It rests on estimating curvature accurately at the interface, regardless of mesh size. Although the level-set framework provides a straightforward relation to calculate curvature, it does not always yield satisfactory approximations when the level-set field lacks sufficient smoothness and regularity. With this in mind, we have recently proposed network-only [45] and hybrid inference systems [46] that tackle the curvature problem in low-resolution grids. Our investigation has revealed that blending traditional schemes with multilayer perceptrons produces better results than taking each component alone. In like manner, Buhendwa et al. [31] have developed a machine-learning interface-reconstruction (IR) strategy that computes volume fractions and apertures. Their procedure has proven effective for under-resolved regions while recovering the conventional IR's accuracy for well-resolved sectors.

Often, artificial mass loss and numerical diffusion occur simultaneously. Further, the coarser the mesh, the more that one loses mass to under-resolution. Consequently, mesh refinement is the preferred option to minimize numerical dissipation. In order to refine a grid efficiently, researchers have proposed nonuniform discretization schemes to increase the resolution only next to the interface [47, 48]. Adaptive Cartesian grids, for instance, have served as the basis for high-order robust level-set algorithms and tools in both serial [49-55] and distributed computing systems $[56,57]$. Over the years, practitioners have also combined level-set technologies with other numerical frameworks to improve mass conservation. Among these hybrid approaches, we can find the coupled level-set and volume-of-fluid [44], the particle level-set [58], the coupled volume-of-fluid and level-set [59], and the marker level-set [60] methods. Other computational scientists have opted for in-place augmentation by either adding volume reinitialization [61] or incorporating source/sink terms [62] into the standard formulation. However, little has been done to investigate mass loss from a data-driven perspective. In this manuscript, we bridge the level-set method with machine learning to offset numerical viscosity and preserve mass in low-resolution grids.

The present study draws inspiration from image super-resolution methodologies. The image super-resolution problem involves learning an end-to-end mapping between low- and high-resolution images [7]. In scientific computing, this concept is strongly connected with training low-resolution models to imitate the rules in their high-resolution counterparts [63, 64]. This idea, in particular, offers promising opportunities to circumvent the prohibitive costs of solving PDEs in grids with considerably small mesh sizes. In computer graphics, for instance, Xie et al. [13] have designed temporally coherent generative adversarial neural networks. Their networks learn to produce volumetric frames from a low-resolution field containing passively advected density and velocity data. Likewise, Liu et al. [65] have reconstructed turbulent flows from coarse spatiotemporal data with the aid of convolutional and multi-scale residuals blocks. Other influential developments in CFD include the learned discretizations of [63] and [64]. In [64], Zhuang et al. extend their data-driven discretizations in [63] to two-dimensional passive scalar advection in turbulent flows. Instead of using predefined finite-volume coefficients, Zhuang et al. have trained convolutional neural networks that emit optimal coefficients based on high-resolution simulations. Their machine learning approach thus keep an exceptional level of accuracy, even when the solution is under-resolved with classic methods. Also, Pathak et al. [66] have developed a PDE-machine-learning strategy to enhance the accuracy of coarse high-Re turbulent-flow simulations. Their framework's key component is an error-correcting U-Net [9] convolutional network. This architecture helps restore fine-scale details in a high-resolution estimate of system variables and simultaneously corrects the error introduced 
during the coarse-grid simulation.

In this manuscript, we introduce a data-driven strategy that extends the notions in [64] and [66] to the level-set method. More precisely, we seek to minimize numerical viscosity in the coarse-mesh evolution of an interface to improve the accuracy of the solution to an FBP. To this end, we consider the semi-Lagrangian formulation [67-69] as our starting point. Semi-Lagrangian schemes are popular in the level-set community because of their unconditional stability and trivial nonuniform-grid implementation. Here, we blend these ordinary schemes with an error-quantifying neural network that corrects the numerically estimated surface trajectory. Unlike [64-66], our model is a plain multilayer perceptron. This network processes localized level-set, velocity, and positional data in a single time frame for select vertices near the moving front. Our main contribution is thus a novel machine-learning-augmented transport algorithm that operates alongside selective redistancing and alternates with standard advection to keep the adjusted interface trajectory smooth. Consequently, our procedure is more efficient than full-scan convolutional-based applications because it concentrates computational effort only around the free boundary. Also, we show through various tests that our strategy is effective at counteracting both numerical diffusion and mass loss. Some of these assessments reveal that our method can achieve the same precision as the baseline scheme at twice the resolution but at a fraction of the cost. Similarly, we prove that our hybrid technique can produce feasible solidification fronts for crystallization processes. In addition, our approach exhibits great generalization for simulation times that exceed the durations employed during training. Together, these assets make our framework attractive to parallel level-set algorithms. Its appeal resides in the possibility of avoiding further mesh refinement and decreasing expensive communications between computing nodes.

We have organized the paper as follows. Sections 2 to 4 describe the level-set method, quadtree Cartesian grids, and semi-Lagrangian advection schemes. Then, we state our methodology and related algorithms for training and deployment in Section 5. After these, Section 6 evaluates our approach through several standard test cases. Finally, we discuss our results, limitations, and possible avenues for future work in Section 7.

\section{The level-set method}

The level-set method [36] is an Eulerian formulation for capturing and tracking interfaces that undergo complex topological changes. The level-set representation denotes an interface by $\Gamma \doteq\{\mathbf{x}: \phi(\mathbf{x})=0\}$, where $\phi(\mathbf{x}): \mathbb{R}^{n} \mapsto \mathbb{R}$ is a higher-dimensional, Lipschitz relation known as the level-set function. In this framework, the zero-isocontour partitions the computational domain $\Omega \subseteq \mathbb{R}^{n}$ into the non-overlapping interior and exterior regions defined by $\Omega^{-} \doteq$ $\{\mathbf{x}: \phi(\mathbf{x})<0\}$ and $\Omega^{+} \doteq\{\mathbf{x}: \phi(\mathbf{x})>0\}$. Then, given some velocity field $\mathbf{u}(\mathbf{x})$, we can evolve $\phi(\mathbf{x})$ and $\Gamma$ by solving the level-set equation:

$$
\phi_{t}+\mathbf{u} \cdot \nabla \phi=0
$$

Assuming that $\phi(\mathbf{x})$ remains sufficiently smooth after advection, one can use the following straightforward expressions to compute normal vectors and mean curvature for any point $\mathbf{x} \in \Omega$ :

$$
\hat{\mathbf{n}}(\mathbf{x})=\frac{\nabla \phi(\mathbf{x})}{\|\nabla \phi(\mathbf{x})\|}, \quad \kappa(\mathbf{x})=\nabla \cdot \frac{\nabla \phi(\mathbf{x})}{\|\nabla \phi(\mathbf{x})\|}
$$

When $\mathbf{u}(\mathbf{x})$ does not depend directly on $\phi(\mathbf{x})$, eq. (1) is linear, and we can solve it with a semi-Lagrangian approach $[67,68]$. Compared with other numerical schemes, the semi-Lagrangian method is unconditionally stable. It is also trivial to implement on adaptive Cartesian grids, whereas higher-order advection solvers are challenging. Combining the semi-Lagrangian formulation with nonuniform meshes is thus convenient because it allows us to solve eq. (1) in level-set applications with very high grid resolutions.

In general, there is an infinite number of functions for which $\Gamma$ describes the same zero level set. Usually, one chooses $\phi(\mathbf{x})$ as a signed distance function because it simplifies computations (e.g., $\|\nabla \phi(\mathbf{x})\|=1$ ) and leads to robust numerical results [70]. Also, signed distance functions are beneficial because they are uniquely determined as the viscosity solutions to the Eikonal equation [71]. Recent studies [45, 46] suggest that these functions can improve the accuracy of machine learning estimations, too. However, as a numerical simulation progresses, a signed distance function can deteriorate quickly, developing noisy features that compromise stability and precision. For this reason, it is customary to redistance $\phi(\mathbf{x})$ frequently by solving the pseudo-time transient reinitialization equation [70]

$$
\phi_{\tau}+\operatorname{sgn}\left(\phi^{0}\right)(\|\nabla \phi\|-1)=0
$$



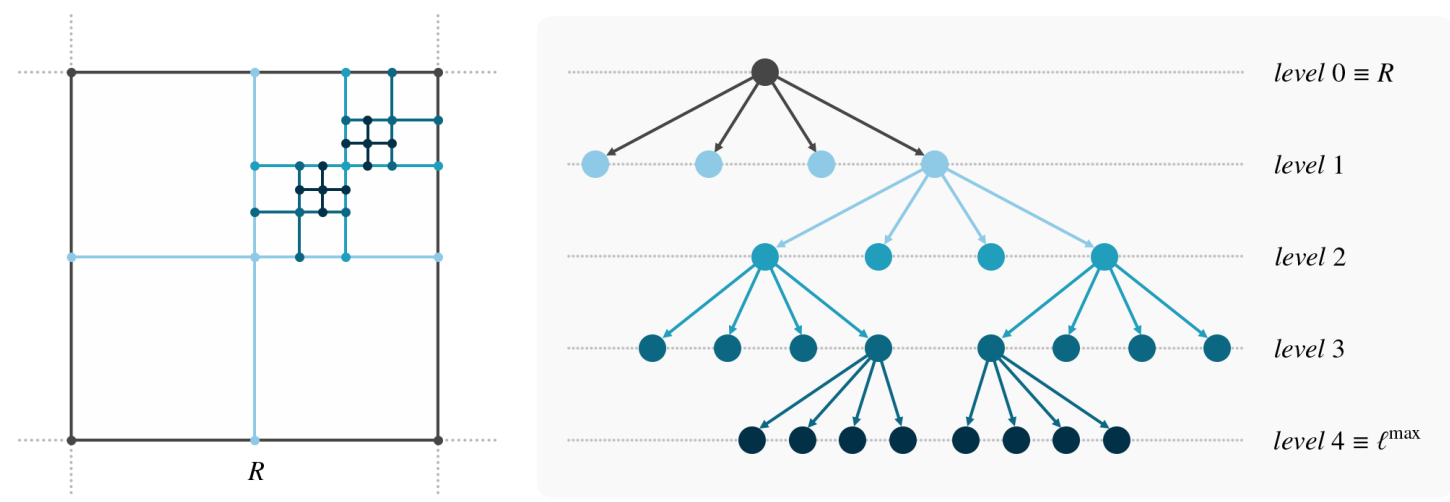

Figure 1: A (non-graded) quadtree Cartesian grid (left) and its data structure representation (right). The quadtree covers a region $R \subseteq \Omega$ corresponding to its root (level 0$)$. We denote grid nodes as small dots at the intersections of the quadtree cells. (Color online.)

where $\tau$ is a pseudo-time stepping variable, $\phi^{0}$ is the starting level-set function, and $\operatorname{sgn}(\cdot)$ is a smoothed-out signum function.

Typically, one uses a TVD Runge-Kutta scheme in time and a Godunov discretization in space to solve eq. (3) to a steady state (i.e., $\phi_{\tau}=0$ and $\|\nabla \phi\|=1$ ). For efficiency, we often stop this iterative process after a prescribed number of steps $v$, depending on the application and how close $\phi^{0}$ is to a signed distance function. For a detailed description of the reinitialization algorithm, we refer the reader to the work of Min and Gibou [51] and Mirzadeh et al. [57]. In both cases, the authors redesigned the redistancing procedure for adaptive Cartesian grids, which we discuss in Section 3. For a complete presentation of the level-set technologies, the reader may consult Osher, Fedkiw, and Sethian's classic texts [72, 73] and the latest review by Gibou et al. [74].

\section{Adaptive Cartesian grids}

Unlike uniform meshes, adaptive Cartesian grids significantly reduce the cost of solving FBPs with level-set methods. They do so by increasing the spatial resolution only close to $\Gamma$, where accuracy is needed the most [48]. In this work, we discretize a computational domain $\Omega$ with the help of standard quadtrees and signed distance level-set functions to digitize the corresponding grid $\mathcal{L}_{\text {. }}$.

A quadtree is a rooted data structure composed of discrete cells covering a rectangular region $R \subseteq \Omega$. A tree cell $C$ has four children (i.e., quadrants) or is a leaf [75]. Also, each cell contains a list with its vertex coordinates, nodal level-set values, and other application-dependent data. Figure 1 illustrates a quadtree with its cells organized into $0 \leqslant L=\ell^{\max }+1$ levels. Given an $L$-level quadtree, we can perform several operations efficiently, like searching in $\mathcal{O}\left(\ln \left(\ell^{\max }\right)\right)$ time and sorting [48]. In addition, we can determine the mesh size or minimum cell width $h$ by establishing a relationship between $\Omega$ 's side lengths and the maximum level of refinement $\ell^{\max }$.

As with most level-set applications based on quadtree Cartesian grids, we have adopted Min's extended Whitney decomposition [76] to refine the mesh near the zero-isocontour. To construct or update a quadtree, we begin at its root $R$ and recursively subdivide its cells according to their distance to $\Gamma$ until we reach level $\ell^{\max }$. In particular, we mark cell $C$ for refinement if the condition

$$
\min _{v \in \operatorname{vertices}(C)}|\phi(v)| \leqslant \operatorname{Lip}(\phi) \cdot \operatorname{diag}(C)
$$

is valid, where $v$ is a vertex, $\operatorname{Lip}(\phi)$ is the level-set function's Lipschitz constant (set to 1.2 ), and $\operatorname{diag}(C)$ is $C$ 's diagonal length. Conversely, we mark any cells for coarsening whenever they cannot fulfill the above criterion [57].

Our research extends the parallel level-set methods provided in [57], which rely on Burstedde and coauthors' p4est library [56]. p4est is a suite of highly scalable routines for refining, coarsening, partitioning, and loadbalancing grids. This library represents the adaptive grid $G$ as a forest of abutting quadtrees rooted in individual cells of a coarse parent grid known as the macromesh. The most important contribution of Mirzadeh et al. in [57] is a parallel interpolation procedure. This algorithm exploits p4est's ghost layering and global nodal indexing by recreating the entire forest as a per-process hierarchical representation. As shown in Section 5, we have employed their local replication and multi-process interpolation mechanisms to realize semi-Lagrangian advection. For more 


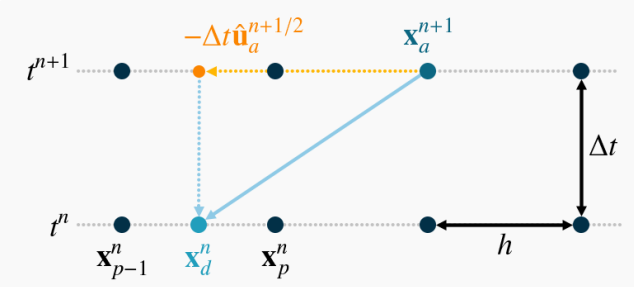

Figure 2: Illustration of backtracking with a one-dimensional semi-Lagrangian scheme. $\mathbf{x}_{a}^{n+1}$ is the arrival grid point, $\mathbf{x}_{d}^{n}$ is its departure point lying between $\mathbf{x}_{p}^{n}$ and $\mathbf{x}_{p-1}^{n}$, and $\hat{\mathbf{u}}_{a}^{n+1 / 2}$ is the intermediate velocity from the midpoint method. The space-time characteristic appears as a light blue solid arrow and maps $\mathbf{x}_{a}^{n+1}$ to $\mathbf{x}_{d}^{n}$. Adapted from [69]. (Color online.)

details about p4est and the level-set methods for distributed-memory systems using efficient MPI communications [77], we refer the reader to their respective manuscripts and handouts.

\section{Semi-Lagrangian advection}

Semi-Lagrangian schemes [68] are extensions of the Courant-Isaacson-Rees method [67]. Practitioners have verified their convergence for several FBPs with passive transport where eq. (1) is hyperbolic. For parabolic PDEs-where the Courant-Friedrichs-Lewy (CFL) condition requires $\Delta t \sim \mathcal{O}\left(h^{2}\right)$ for most explicit methods-semi-Lagrangian schemes can also converge with $\Delta t \sim \mathcal{O}(h)$ if one integrates frequent reinitialization and velocity smoothing [48].

Because of their versatility, unconditional stability, and ease of implementation, practitioners have resorted to semi-Lagrangian methods for solving FBPs in both uniform and adaptive grids. Semi-Lagrangian schemes, however, are not conservative and often lead to mass loss when applied to the evolution of the level-set function [74].

The basis of this numerical technique is that we begin with an Eulerian mesh with known grid points and level-set values at the current time step. Also, we know the vertex locations in the next time frame, but we must solve for the unknown new level-set values at these points [69]. To approximate the solution to eq. (1) at these locations, we can integrate the system

$$
\left\{\begin{aligned}
\frac{\mathrm{d} X(t)}{\mathrm{d} t} & =\mathbf{u}(X(t)) \\
\frac{\mathrm{d} \phi(X(t), t)}{\mathrm{d} t} & =0
\end{aligned}\right.
$$

along the characteristic curves $X(t)$ backward in time. Thus, if $\mathcal{L}^{n+1}$ is the computational grid at time $t^{n+1}=t^{n}+\Delta t,{ }^{1}$ we start at a known arrival grid point $\mathbf{x}_{a}^{n+1}$ lying on the trajectory $X^{n+1}$ and trace it back to its departure point $\mathbf{x}_{d}$ in the upwind direction. Then, we compute its level-set value as in [57] with

$$
\phi^{n+1}\left(\mathbf{x}_{a}^{n+1}\right)=\phi^{n+1}\left(X^{n+1}\right)=\phi\left(X\left(t^{n+1}\right), t^{n+1}\right)=\phi\left(X\left(t^{n}\right), t^{n}\right)=\phi^{n}\left(X^{n}\right)=\phi^{n}\left(\mathbf{x}_{d}\right),
$$

where the second-order accurate midpoint method provided in $[51,78]$,

$$
\begin{aligned}
\hat{\mathbf{x}} & =\mathbf{x}_{a}^{n+1}-\frac{\Delta t}{2} \mathbf{u}^{n}\left(\mathbf{x}_{a}^{n+1}\right) \\
\mathbf{x}_{d} & =\mathbf{x}_{a}^{n+1}-\Delta t \mathbf{u}^{n+\frac{1}{2}}(\hat{\mathbf{x}}),
\end{aligned}
$$

locates the departure point using the intermediate velocity

$$
\mathbf{u}^{n+\frac{1}{2}}=\frac{3}{2} \mathbf{u}^{n}-\frac{1}{2} \mathbf{u}^{n-1} .
$$

\footnotetext{
${ }^{1}$ We use the traditional shorthand temporal discretization $\psi^{n} \equiv \psi\left(t^{n}\right)$ for variable $\psi$.
} 
Since $\hat{\mathbf{x}}$ and $\mathbf{x}_{d}$ do not necessarily coincide with grid points, we must interpolate the appropriate fields from $\mathcal{L}^{n}$ and $\mathcal{G}^{n-1}$ to calculate $\mathbf{u}^{n+\frac{1}{2}}(\hat{\mathbf{x}})$ and $\phi^{n}\left(\mathbf{x}_{d}\right)$. Figure 2 shows this situation for a one-dimensional semi-Lagrangian scheme. In this work, we employ bilinear and quadratic interpolation to sample scalar and vector fields on quadtree Cartesian grids. As detailed by Strain [48], if $C \in \mathcal{G}_{\mathcal{L}}$ is an $h$-by- $h$ quadtree cell with lower-left coordinates $\left(x_{0}, y_{0}\right)$, we can calculate $\phi(\mathbf{x})$ at $(x, y)=\left(x_{0}+\alpha h, y_{0}+\beta h\right) \in C$ using the piecewise bilinear interpolation form

$$
\phi(x, y)=(1-\alpha)(1-\beta) \phi_{00}+(1-\alpha)(\beta) \phi_{01}+(\alpha)(1-\beta) \phi_{10}+(\alpha)(\beta) \phi_{11},
$$

where $\phi_{i j}=\phi\left(x_{0}+i h, y_{0}+j h\right)$ is a vertex value in $C$. Similarly, as noted by Min and Gibou [51], we can get a quadratic interpolation procedure by correcting eq. (9) with second-order derivatives. Hence,

$$
\begin{aligned}
\phi(x, y)= & (1-\alpha)(1-\beta) \phi_{00}+(1-\alpha)(\beta) \phi_{01}+(\alpha)(1-\beta) \phi_{10}+(\alpha)(\beta) \phi_{11} \\
& -h^{2} \frac{(\alpha)(1-\alpha)}{2} \phi_{x x}-h^{2} \frac{(\beta)(1-\beta)}{2} \phi_{y y}
\end{aligned}
$$

where $\phi_{x x}$ and $\phi_{y y}$ are bilinearly interpolated at $(x, y)$ from their corresponding vertex values for $C$. The reader may consult [51] for more information about the standard finite-difference formulations to discretize $\phi_{x x}$ and $\phi_{y y}$ on nongraded, adaptive meshes. The textbook by [69] also provides a detailed overview of semi-Lagrangian schemes and their applications. In the present study, we have instrumented eqs. (9) and (10) within Algorithm 2 in [57] for heterogeneous computing systems.

\section{Methodology}

In this section, we state our data-driven strategy to improve the accuracy of semi-Lagrangian schemes in quadtree Cartesian grids. Our approach blends machine learning with the level-set technologies of [51] and [57] to solve a specialized instance of the image super-resolution problem [7]. The idea behind our framework stems from the fact that highly resolved grids yield much better solutions to FBPs than their coarser counterparts. Here, as in [66], we investigate the possibility of computing on-the-fly corrections for a coarse-grid interface trajectory so that it closely follows its corresponding evolution in a finer mesh.

\subsection{Problem definition}

Let $\mathcal{S}^{n}$ be the state of the solution to an FBP at time $t^{n}$. $\mathcal{S}$ embodies a computational grid besides its nodal levelset, velocity, pressure, and temperature values, among others. Further, define FBPEqnSolver() as a sequence of nonlinear operators acting on $\delta^{n}$. FBPEqnSolver () prepares the advection of $\phi^{n} \in \delta^{n}$ by producing the intermediate state $\hat{\mathcal{S}}^{n}=$ FBPEqnSolver $\left(\mathcal{S}^{n}\right)$. Then, given a time step $\Delta t$ and a SemiLagrangian() procedure that performs the operations described in Section 4, the statement

$$
\left.\delta^{n+1}=\text { SemiLagrangian (FBPEqnSolver }\left(\delta^{n}\right), \Delta t\right)
$$

finalizes the solution at time $t^{n+1}$. In particular, the SemiLagrangian() method arrives at $\mathcal{S}^{n+1}$ by advancing the sampled level-set function from $\phi^{n}$ to $\phi^{n+1}$. Also, it updates the underlying quadtree Cartesian mesh from $\mathcal{G}^{n}$ to $\mathcal{G}^{n+1}$. For a technical description of the SemiLagrangian() subroutine, the reader may consult Algorithm 3 in [57].

Next, consider a simulation that starts by discretizing $\Omega$ with a coarse mesh $\mathscr{L}_{c}^{0}$ and a fine mesh $\mathscr{L}_{f}^{0}$, where $\ell_{c}^{\max }<$ $\ell_{f}^{\max }$, and $h_{c}>h_{f}$ (see fig. 1) ${ }^{2}$. In addition, assume that the nodal coordinates in $\mathcal{L}_{c}^{n}$ are a proper subset of the vertex coordinates in $\mathcal{L}_{f}^{n}$ for all $t^{n}$. If $\mathcal{S}_{c}\left(t^{0}\right)=\mathcal{S}_{f}\left(t^{0}\right)$ is the FBP's initial condition ${ }^{3}$ involving the simultaneous coarse- and fine-grid solutions, then

$$
\mathcal{S}_{c}\left(t^{0}+\delta\right)=\operatorname{SemiLagrangian}\left(\hat{\mathcal{S}}_{c}^{0}, \delta\right) \neq \operatorname{SemiLagrangian}\left(\hat{\mathcal{S}}_{f}^{0}, \delta\right)=\mathcal{S}_{f}\left(t^{0}+\delta\right)
$$

after some finite time interval $\delta>0$. More precisely, $\phi_{c}\left(t^{0}+\delta\right) \neq \phi_{f}\left(t^{0}+\delta\right)$, and $\Gamma_{c}\left(t^{0}+\delta\right) \neq \Gamma_{f}\left(t^{0}+\delta\right)$, mainly because of the numerical diffusion in the SemiLagrangian() function. Consequently, both the coarse- and the fine-grid solutions will diverge as the simulation progresses.

\footnotetext{
${ }^{2}$ Unless otherwise stated, the subscript $c$ refers to a coarse-grid feature/component and $f$ to the fine-grid counterpart.

${ }^{3}$ In practice, it suffices that $\delta_{c}\left(t^{0}\right)$ and $\delta_{f}\left(t^{0}\right)$ are approximately equal along a narrow region of interest (e.g., in a shell around $\left.\Gamma_{c}^{0}\right)$.
} 


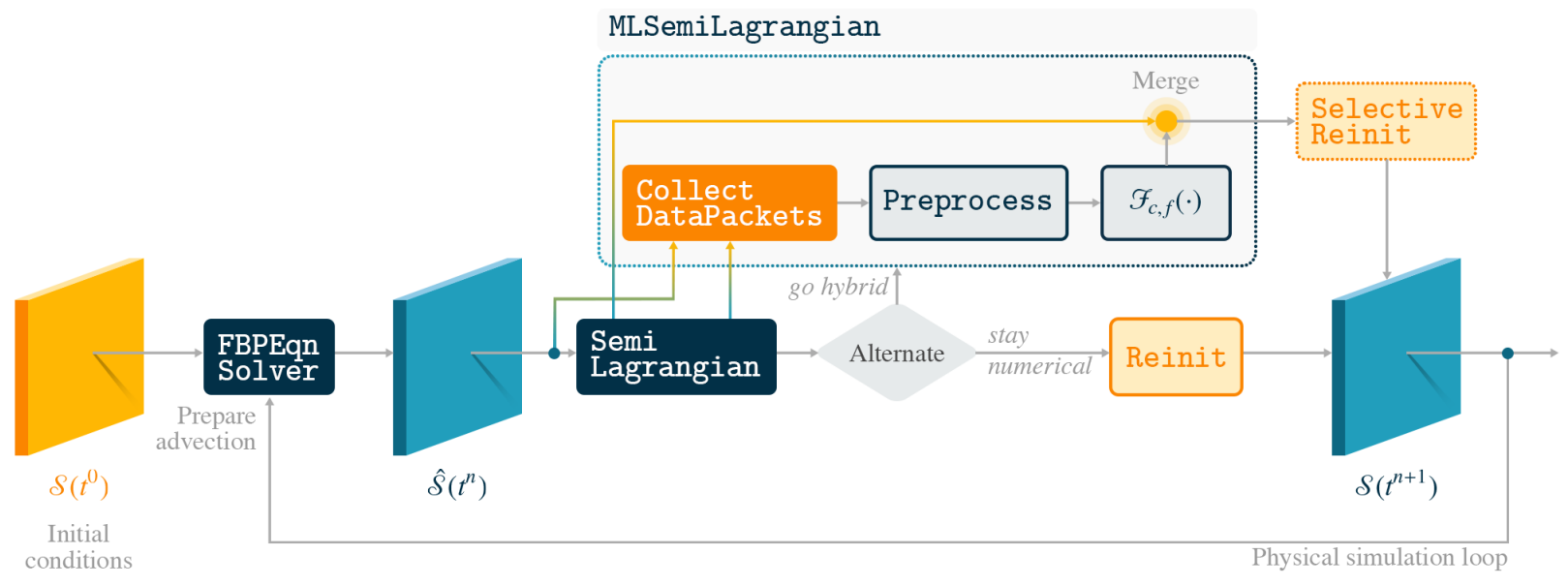

Figure 3: Overview of our hybrid semi-Lagrangian solver. The MLSemiLagrangian () routine couples numerical advection with the errorcorrecting neural network $\mathscr{F}_{c, f}(\cdot)$ to improve the level-set accuracy of grid points next to $\Gamma^{n}$. A full description of MLSemiLagrangian() is provided in Algorithm 1. Our solver alternates between standard transport and machine-learning-induced advection. When using the neural model, we apply selective reinitialization to protect the level-set values in a subset of the corrected trajectory. The alternating mechanism helps smooth out the level-set values near the evolving front since the MLSemiLagrangian() module cannot guarantee regularity on its own. (Color online.)

In this study, if $\bar{\delta} \sim \mathcal{O}\left(h_{c}\right)$ is a small time interval and the velocity field satisfies $\max \left\|\mathbf{u}_{c}(\mathbf{x})\right\|=1, \forall \mathbf{x} \in \Omega$, we claim that one can characterize $\delta_{c}\left(t^{0}+\bar{\delta}\right)$ 's deviation from $\delta_{f}\left(t^{0}+\bar{\delta}\right)$ with

$$
\phi_{f}\left(t^{0}+\bar{\delta}\right)=\phi_{c}\left(t^{0}+\bar{\delta}\right)+\bar{\varepsilon}
$$

where $\bar{\varepsilon}$ is the coarse level-set error.

Therefore, if we can model $\bar{\varepsilon}$ in eq. (13) at regular intervals $\bar{\delta}$, we will be able to correct $\delta_{c}$, improve its precision, and reduce artificial mass loss. As introduced in Section 3, we can further simplify this error model by concentrating our effort only at vertices next to $\Gamma$, where accuracy is critical.

\subsection{Error-correcting neural networks for semi-Lagrangian advection}

Our goal is to design a function that quantifies the local error $\bar{\varepsilon}$ incurred by $\phi_{c}^{n}(\mathbf{x})$ 's semi-Lagrangian transport for any vertex next to the interface. In this model, we should leverage the statistical information available from various fields in $\hat{\delta}_{c}^{n}$ in the neighborhood of $\Gamma_{c}^{n}$. Upon evaluating these fields, our function should estimate (with high precision) how much numerical dissipation in $\phi_{c}^{n+1}(\mathbf{x})$ has taken place as we advanced the simulation from $t^{n}$ to $t^{n+1}$.

Let $\mathscr{F}_{c, f}(\cdot)$ be our error-correcting function for advected level-set values near $\Gamma_{c}^{n}$. Here, we follow state-of-theart machine learning approaches in CFD [13, 64-66] and materialize $\mathscr{F}_{c, f}(\cdot)$ as a neural network. More specifically, we resort to image super-resolution methodologies to estimate $\bar{\varepsilon}$ and construct $\mathscr{F}_{c, f}(\cdot)$ by abstracting the difference between the coarse-mesh semi-Lagrangian advection of $\phi_{c}^{n}(\mathbf{x})$ and the numerical reference trajectory in a fine grid.

Figure 3 describes how $\mathscr{F}_{c, f}(\cdot)$ interacts with a conventional FBP solver. The MLSemiLagrangian() block is the main contribution of this research. First, this module gathers data packets from intermediate state information and SemiLagrangian()-advected level-set values for grid nodes near the interface at time $t^{n}$. Next, a custom subroutine preprocesses these packets and routes them to $\mathscr{F}_{c, f}(\cdot)$ for evaluation. Finally, $\mathscr{F}_{c, f}(\cdot)$ yields improved local estimations to $\phi_{c}^{n+1}(\mathbf{x})$ that are subsequently re-integrated to the SemiLagrangian() computations. The advection algorithm within the MLSemiLagrangian() method is iterative and involves successive mesh coarsening and refining steps until $\mathcal{G}_{c}^{n+1}$ converges (see Section 3.2 in [57]). For efficiency, we cache the machine-learning-corrected level-set values from the first iteration. Then, we merge them with the proposed numerical estimations before each re-gridding operation. In addition to the MLSemiLagrangian() unit, our framework includes a selective reinitialization function that masks out and protects a portion of the enhanced trajectory from the perturbations arising when solving eq. (3). As in [57], we integrate eq. (3) using a second-order accurate TVD-RK scheme [79] with explicit, adaptive time-stepping and a Godunov discretization with quadratic interface localization in space [51]. Figure 3 also depicts an alternating mechanism between standard advection and machine-learning-induced transport. Such a mechanism is fundamental to (1) counteract the lack of physical constraints in $\mathscr{F}_{c, f}(\cdot)$ and (2) regularize the adjusted level set values next to $\Gamma_{c}^{n}$. The following sections detail how all these elements interplay within our hybrid advection system. 


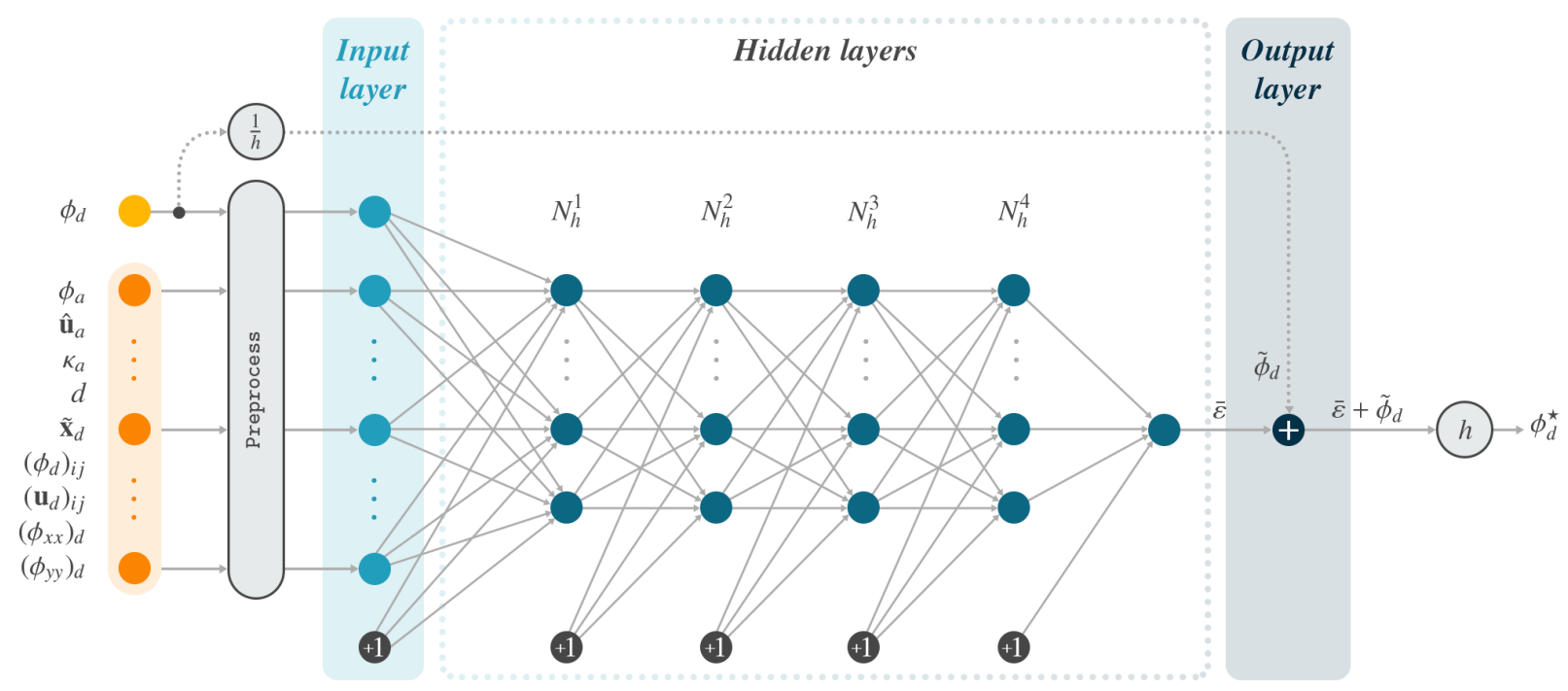

Figure 4: The error-correcting neural network $\mathscr{F}_{c, f}(\cdot)$ employed in the MLSemiLagrangian() module of fig. 3. We also show the Preprocess () subroutine and the normalization/denormalization operations for the level-set value $\phi_{d}$ estimated with the semi-Lagrangian scheme of Section 4 . In general, we expect the corrected output $\phi_{d}^{\star}$ to be closer to the level-set value computed with a fine grid. (Color online.)

Our neural model resembles a localized version of the solver in [66] and adapts the hybrid strategy in [64] to the level-set framework. Figure 4 outlines $\mathscr{F}_{c, f}(\cdot)$ 's architecture. Unlike [64, 66], $\mathscr{F}_{c, f}(\cdot)$ is an ordinary multilayer perceptron that outputs a corrected level-set value after digesting a small input vector. Besides a typical feedforward architecture for estimating $\bar{\varepsilon}, \mathscr{F}_{c, f}(\cdot)$ features a skip connection that carries the $h$-normalized ${ }^{4}$ level-set value $\tilde{\phi}_{d}$ to a non-trainable additive neuron that computes $\bar{\varepsilon}+\tilde{\phi}_{d}$. In the end, upon applying $h$-denormalization (subsumed in $\mathscr{F}_{c, f}(\cdot)$ 's box in fig. 3), we produce a better level-set approximation, $\phi_{d}^{\star}$, for advancing the moving front. Compared to [65], $\mathscr{F}_{c, f}(\cdot)$ exploits only low-resolution data from one step, thus relieving the hybrid solver from buffering temporal information beyond time $t^{n}$. Figure 4 also displays the preprocessing module, which transforms incoming data according to patterns observed during training. Similar to our findings in [46], the Preprocess () subroutine is crucial for the MLSemiLagrangian() procedure because it favors learning convergence and increases $\mathscr{F}_{c, f}(\cdot)$ 's accuracy. We discuss the Preprocess() building block alongside its technical implementation in Section 5.4.2.

Our goal is to ensure that the free boundary motion from $\Gamma^{n}$ to $\Gamma^{n+1}$ is highly accurate. For this reason, we reserve $\mathscr{F}_{c, f}(\cdot)$ for data packets collected for points $\mathbf{x}_{a}^{n+1}$ lying next to the known interface at time $t^{n}$. As shown in fig. 4, the corresponding input for a qualified vertex $\mathbf{x}_{a}^{n+1}$ to $\mathscr{F}_{c, f}(\cdot)$ is a preprocessed version of data available to the SemiLagrangian() subroutine. The latter is the basis to compute $\phi^{n+1}\left(\mathbf{x}_{a}^{n+1}\right)=\phi^{n}\left(\mathbf{x}_{d}\right)$. Among these data, we find level-set values, distances, velocity components, and second-order derivatives. All of them take the role of arguments in eqs. (6) to (10). We will describe the data-extraction procedure later in Section 5.3.

In order to specify a data packet formally, suppose we trace a vertex located at $\mathbf{x}_{a}$ near $\Gamma^{n}$ back to its departure point $^{5} \mathbf{x}_{d}$. To do so, we move in the $-\Delta t \hat{\mathbf{u}}_{a}$ direction according to eq. (7), where $\hat{\mathbf{u}}_{a} \equiv \mathbf{u}(\hat{\mathbf{x}}) \equiv \mathbf{u}^{n}(\hat{\mathbf{x}})$. This last expression holds because we assume that $\mathbf{u}^{n+\frac{1}{2}} \equiv \mathbf{u}^{n}$ in eq. (8). In particular, we can find $\hat{\mathbf{x}}$ using only the velocity field at $t^{n}$ and eq. (7a) followed by a quadratic interpolation step to evaluate $\mathbf{u}^{n}(\hat{\mathbf{x}})$. Then, we can readily compute $\mathbf{x}_{d}$ with eq. (7b). Since $\mathscr{F}_{c, f}(\cdot)$ does not consume entries from previous solution states, from now on, we will drop the $n$ superscript to simplify the notation.

Next, let $C$ be the $h$-by- $h$ square cell at the maximum level of refinement that owns $\mathbf{x}_{d}$. From $C$, we can build a data packet

\footnotetext{
${ }^{4}$ We indicate $h$-normalized variables with a tilde $\sim$ accent.

${ }^{5}$ For data-packet specific attributes, we use the subscript $a$ to denote information at the arrival point and $d$ for the departure point.
} 


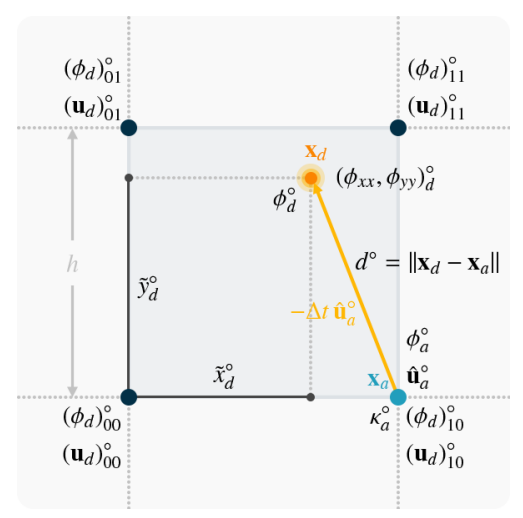

(a) Original data packet $p^{\circ}$

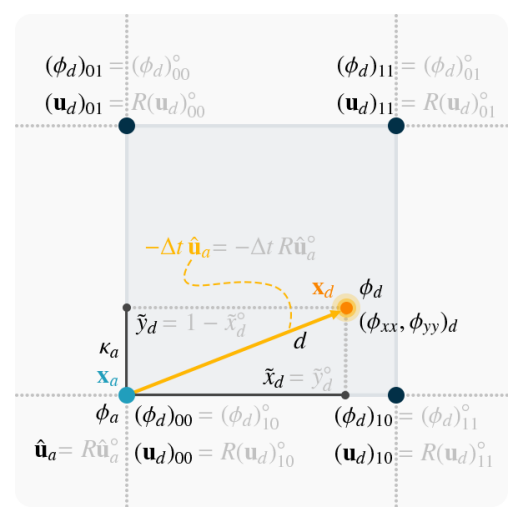

(b) Reoriented data packet $p$ (standard form)

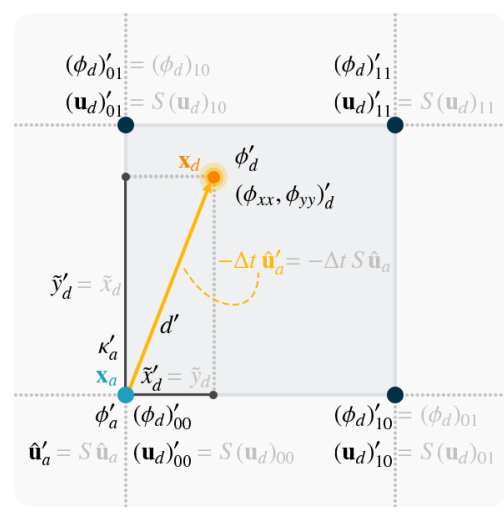

(c) Augmented data packet $p^{\prime}$

Figure 5: An $h$-by- $h$ grid cell $C$ at the maximum level of refinement owning the departure point $\mathbf{x}_{d}$ (in orange) for vertex $\mathbf{x}_{a}$ (in light blue) next to $\Gamma$. Original sampled data from a numerical simulation appears in (a), denoted with a o superscript. Our inference system digests only numerical values shown in black. After reorientation, we obtain the machine-learning-suitable data packet in (b), where $R$ is a rotation transformation (assuming that $\kappa_{a}^{\circ}<0$ ). Invariant data to rotation transfers directly from (a) to (b), and we do not indicate any equality explicitly (e.g., $\phi_{d}=\phi_{d}^{\circ}$ ). (c) illustrates data augmentation by reflection about the line $y=x+\beta$, with $\beta \in \mathbb{R}$, going through $\mathbf{x}_{a}$. A permutation matrix $S$ swaps the elements of multicomponent reoriented data in (b) (e.g., velocities). Invariant data to reflection transfers directly from (b) to (c) (e.g., $\left.\left(\phi_{d}\right)_{00}^{\prime}=\left(\phi_{d}\right)_{00}\right)$. We denote augmented data with a prime superscript. (Color online.)

$$
p=\left(\begin{array}{rl}
\phi_{a}: & \text { level-set value at } \mathbf{x}_{a} \\
\hat{\mathbf{u}}_{a}: & \text { midpoint velocity at } \hat{\mathbf{x}} \\
d: & \text { distance from } \mathbf{x}_{d} \text { to } \mathbf{x}_{a} \\
\left(\tilde{x}_{d}, \tilde{y}_{d}\right): & \mathbf{x}_{d} \text { coordinates w.r.t. }\left(x_{0}, y_{0}\right) \\
\left(\phi_{d}\right)_{i j}: & \text { nodal level-set values }
\end{array}\right.
$$

$\left.\begin{array}{rl}\left(\mathbf{u}_{d}\right)_{i j}: & \text { nodal velocities } \\ \left(\phi_{x x}\right)_{d}: & \left|\partial^{2} \phi / \partial x^{2}\right| \text { bilinearly interpolated at } \mathbf{x}_{d} \\ \left(\phi_{y y}\right)_{d}: & \left|\partial^{2} \phi / \partial y^{2}\right| \text { bilinearly interpolated at } \mathbf{x}_{d} \\ \kappa_{a}: & \text { curvature bilinearly interpolated at } \Gamma \\ \phi_{d}: & \text { numerical level-set value at } \mathbf{x}_{d}\end{array}\right) \in \mathbb{R}^{22}$,

where $\left(\psi_{d}\right)_{i j}=\left(\psi_{d}\right)\left(x_{0}+i h, y_{0}+j h\right)$ is a vertex value, $i, j \in\{0,1\}$, and $\left(x_{0}, y_{0}\right)$ is $C$ 's lower-left corner.

Figure 5a describes a data packet in a spatial context, where a $\circ$ superscript differentiates the original from the standard form suitable for machine learning. Figure 5a also shows that $\mathbf{x}_{a}$ always coincides with one of $C$ 's vertices if we consider a unit $C F L$ constant and a velocity maximum-unit-norm restriction. The previous statement about $\mathbf{x}_{a}$ is true regardless of grid resolution and is essential for narrowing the problem at hand.

In earlier research [46], we exploited symmetry in the curvature problem to facilitate the neural-network design and data-set composition. In particular, we confirmed that training only on the negative curvature spectrum favored efficiency, reduced architectural complexity, and accelerated learning. These benefits hinged on available, inexpensive mechanisms to gauge a stencil's convexity and flip the sign of level-set values and inferred curvature accordingly. Here, we rely on this strategy to simplify $\mathscr{F}_{c, f}(\cdot)$ 's topology while preserving at least the same expressive power as a full-curvature-spectrum model. Thus, the idea is to calculate $\kappa_{a}^{\circ}$ in fig. $5 \mathrm{a}$ by discretizing eq. (2) using finite differences. Then, we estimate curvature at

$$
\mathbf{x}_{a}^{\Gamma}=\mathbf{x}_{a}-\phi\left(\mathbf{x}_{a}\right) \frac{\nabla \phi\left(\mathbf{x}_{a}\right)}{\left\|\nabla \phi\left(\mathbf{x}_{a}\right)\right\|}
$$

with bilinear interpolation. $\mathbf{x}_{a}^{\Gamma}$ is a rough approximation for $\mathbf{x}_{a}$ 's projection onto $\Gamma^{n}$. Next, we perform negativecurvature normalization by negating $p^{\circ}$ 's level-set values if $\kappa_{a}^{\circ}>0$. After this step, we proceed to the last preparations before leaving the data packet in a well-suited form for the Preprocess () module. Finally, upon post-processing $\mathscr{F}_{c, f}(\cdot)$ 's output in fig. 4 , we check $\kappa_{a}^{\circ}$ and restore the sign for the machine-learning-corrected level-set value.

We have also resorted to sample reorientation to promote neural learning. This technique helps minimize feature variations and has proven effective in elementary computer vision applications, such as face recognition with eigenfaces $[4,80]$. Figure $5 \mathrm{~b}$ shows a data packet $p$ after reorienting the original $p^{\circ}$, assuming that $\kappa_{a}^{\circ}<0$. Standard-forming $p^{\circ}$ requires rotating its stencil until the angle between the horizontal axis and $-\hat{\mathbf{u}}_{a}$ lies between 0 and $\pi / 2$. Reorientation does not change intrinsic level-set values, curvature, and distance between $\mathbf{x}_{a}$ and $\mathbf{x}_{d}$; however, it affects vector quantities by pre-multiplying them with a rotation transformation $R(\theta)$, where $\theta= \pm k \pi / 2$, and $k \in\{0,1,2\}$. After 
reorientation, the arrival point becomes the origin of a local coordinate system that contains all the information we need in its first quadrant.

Following our description of the error-correcting multilayer perceptron, we present the MLSemiLagrangian() procedure in Algorithm 1 next. This algorithm combines $\mathscr{F}_{c, f}(\cdot)$ with the parallel semi-Lagrangian schemes in [57]. Its formal parameters ${ }^{6}$ include $\mathscr{F}_{c, f}(\cdot)$, the quadtree data structure $\mathcal{G}^{n} \in \hat{\delta}^{n}$, and the scalar-/vector-field vertex values necessary to construct the neural network inputs and advance the moving front. Furthermore, Algorithm 1 assumes that we have optimized $\mathscr{F}_{c, f}(\cdot)$ for reducing numerical diffusion in the advected level-set field for nodes near $\Gamma^{n}$ in a mesh with an $\ell_{c}^{\max }$ maximum level of refinement. Similar to its numerical counterpart, the MLSemiLagrangian() method produces new level-set values and updates the adaptive mesh. In addition, it generates an array of vertex coordinates that we should protect during selective reinitialization. As outlined above, our goal is to output nodal level-set values in $\phi^{n+1}$ with coordinates in $e^{n+1}$, closely resembling the free boundary motion in a higher-resolution grid.

Remark 1. We provide Algorithm 1 and the rest of the routines in the most general way. That is, we have used conventional formulae to determine the time step $\Delta t$ based on a user-defined $C F L$ constant and a possibly unbounded velocity field over $\Omega$. However, to validate our assumptions and narrow down the learning problem, we have set

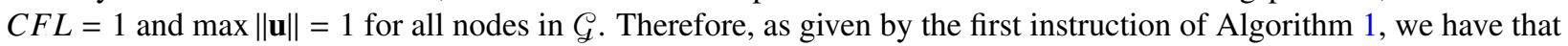
$\Delta t=h$, and we can thus recover the data characterization provided in fig. 5 .

The first step to incorporate machine learning into semi-Lagrangian advection in Algorithm 1 is to collect data packets in $\mathscr{P}$ and their coordinates in $C$ for vertices next to $\Gamma^{n}$. To do so, we get the local hierarchical reconstruction $\mathscr{H}^{n}$ and retrieve the information in eq. (14) and fig. 5a by calling CollectDataPackets (). We discuss CollectDataPackets() in some detail in Section 5.3. Then, we apply negative-curvature normalization and reorientation for each data packet $p \in \mathscr{P}$ to arrive at the standard form referenced in fig. $5 \mathrm{~b}$. At the same time, we populate the ancillary array $\mathcal{S}_{\kappa}$ to record whether $\mathbf{x}_{a}$ is proximal to a concave or convex interface region. For every machine-learning-suitable packet, we generate two samples: one with the preprocessed standard blob $p$ and another with the augmented data packet $p^{\prime}$. For example, fig. 5c illustrates the reflected data packet for the reoriented $p$ shown in fig. $5 \mathrm{~b}$. The rationale for double-sampling is that the departure point's level-set value does not change if we reflect its blob about a slope-one, straight line going through the arrival point. By leveraging this invariance, we can increase the accuracy of $\phi_{d}^{\star}$ if we average the predictions for the corresponding input samples

$$
\left[\operatorname{Preprocess}(p, h), \frac{1}{h} p \cdot \phi_{d}\right] \text { and }\left[\operatorname{Preprocess}\left(p^{\prime}, h\right), \frac{1}{h} p^{\prime} . \phi_{d}\right] \text {. }
$$

Notice that these samples are two-part input vectors that match $\mathscr{F}_{c, f}(\cdot)$ 's expected format. Recently, practitioners have shown that such symmetry-preserving transformations and succeeding averaging can improve machine-learning IR in the level-set method [31]. Here, we have realized these transformations with a few permutations over $p$ 's vertex values.

For efficiency, we accommodate all the samples in matrix $B$. Then, we launch $\mathscr{F}_{c, f}(\cdot)$ in batch mode to predict the $h$-normalized, error-corrected level-set values for the columns in $B$. Upon $h$-denormalization, averaging, and sign-restoration using $\delta_{\kappa}$, we recover the improved $\phi_{d}^{\star}$. Even though we expect $\phi_{d}^{\star}$ to be more accurate than $\phi_{d}$, outlying input patterns might lead to wild predictions in some scenarios. To account for these cases, we revert to $\phi_{d}$ whenever $\mathscr{F}_{c, f}(\cdot)$ predicts $\Gamma^{n}$ should move by more than $h$ or if the $h$-relative difference between $\phi_{d}$ and $\phi_{d}^{\star}$ is over $15 \%$. Neural network divergence is not uncommon in numerical experiments, and we have reported extreme cases when approximating mean curvature [46]. In this research, we mitigate such a vulnerability by taking simple precautions.

While collecting $\phi_{d}^{\star}$, we simultaneously build a hash map $m_{\phi}$ that links nodal coordinates to machine-learningcomputed level-set values. $m_{\phi}$ works as a cache that prevents calling $\mathscr{F}_{c, f}(\cdot)$ more than once. Also, it is essential for data synchronization across processes and sharing what locally aware nodal $\phi_{d}^{\star}$ values should persist after re-gridding. To instrument this map and update the ghost layer, we have used MPI [77], PETSc vectors [81], and the infrastructure in p4est[56].

The concluding part of Algorithm 1 merges the machine learning corrections with the discrete level-set function advected numerically in the rest of the mesh. The coupling occurs in an iterative process based on Algorithm 3 in [57]. In each iteration, we compute the departure points $X_{d}$ and their level-set values $\phi^{n+1}$ using eqs. (6) to (8) and

${ }^{6}$ For consistency, we represent one-element nodal variables as $m$-vectors in lowercase bold faces (e.g., $\boldsymbol{\phi}^{n}$ ) and variables with $d>1$ values per node as $d$-by- $m$ matrices in caps (e.g., $U^{n}$ ). In the p4est terminology, $m$ is the number of independent vertices that $\mathcal{G}^{n}$ is aware of. 


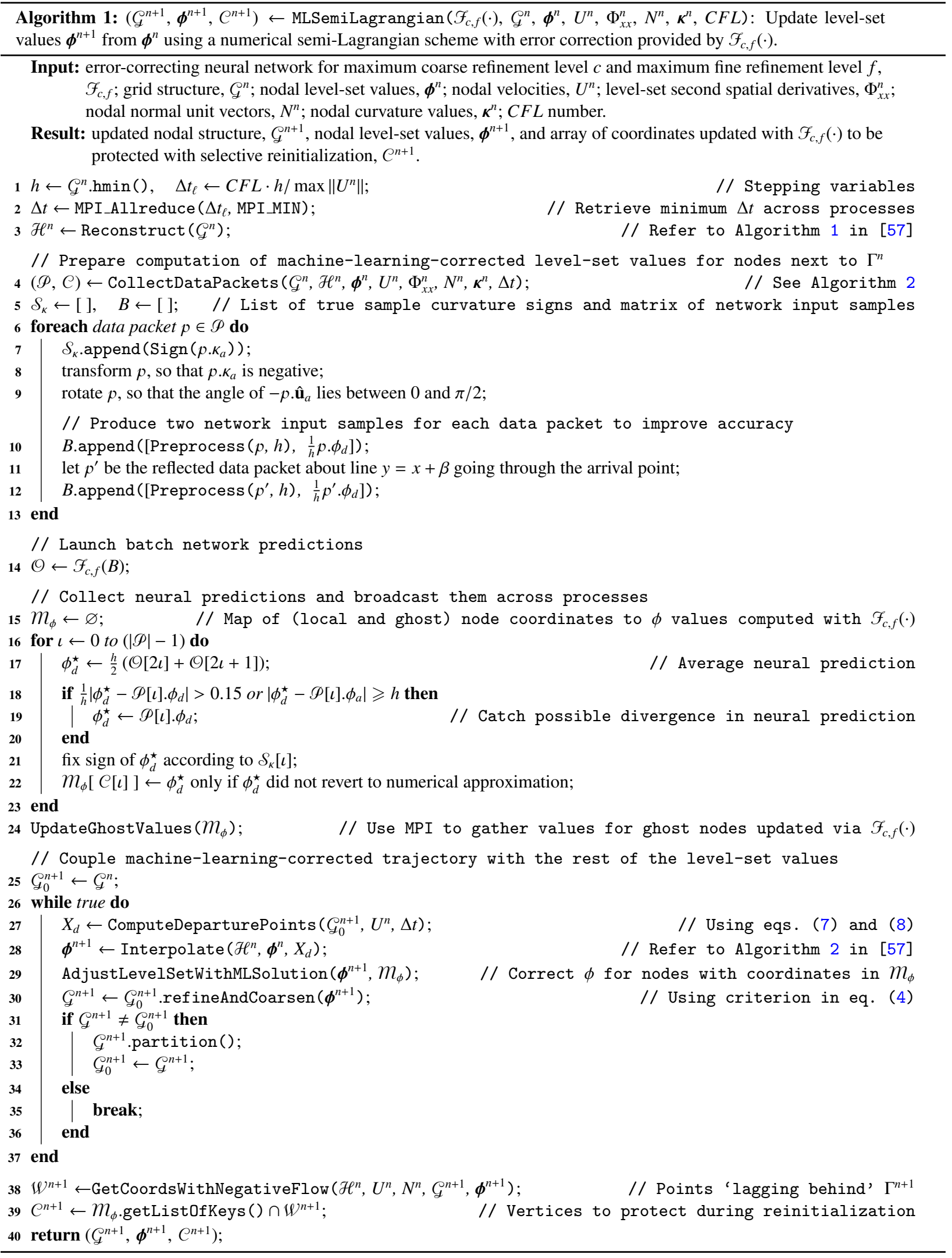




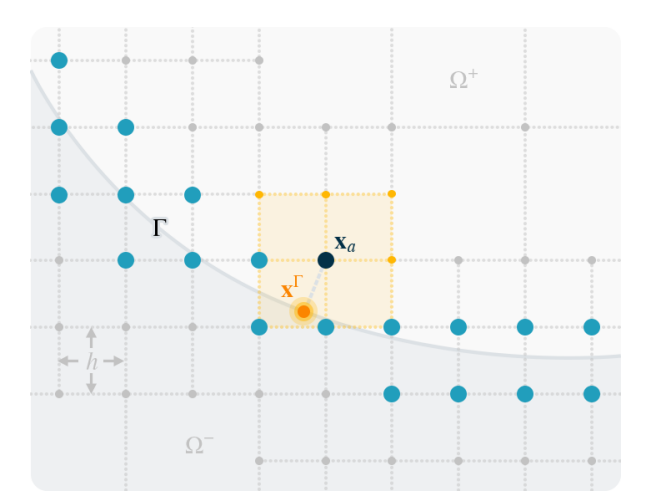

Figure 6: Sampled nodes adjacent to a concave interface region in a quadtree mesh. Vertices with $h$-uniform nine-point stencils appear in light blue. We gather these nodes with the GetLocalNodesNextToGamma() subroutine inside Algorithm 2. Among them, we shade in yellow the stencil of the (arrival) grid point $\mathbf{x}_{a}$ colored in dark blue. The rough estimation to the closest point on $\Gamma$ to $\mathbf{x}_{a}$ is $\mathbf{x}^{\Gamma}$ (shown in orange). (Color online.)

interpolation from data at time $t^{n}$. The Interpolate() method entails a series of distributed actions that rely on synchronization and $\mathscr{H}^{n}$ to retrieve scattered information from different sites. Then, we replace the numerical level-set values with the machine-learning ones in $m_{\phi}$ for independent nodes whose coordinates match with a key in the cache. Afterward, we refine and coarsen the new grid to produce $\mathcal{L}^{n+1}$ according to the criterion in eq. (4). We execute these operations repeatedly until $\mathscr{L}^{n+1}$ converges. Usually, convergence requires no more steps than the maximum number of subdivisions in any quadtree [57].

Upon exiting the update loop, we gather the vertex coordinates in $m_{\phi}$ and intersect them with $\mathfrak{W}^{n+1}$, which results from calling GetCoordsWithNegativeFlow(). This ancillary subroutine locates vertices within a narrow band of half-width $2 h \sqrt{2}$ around $\Gamma^{n+1}$, where the angular condition

$$
\operatorname{acos}\left(-\operatorname{sign}\left(\phi^{n+1}(\mathbf{x})\right) \hat{\mathbf{n}}^{n}(\mathbf{x}) \cdot \hat{\mathbf{u}}^{n}(\mathbf{x})\right) \leqslant \theta_{\mathfrak{W}}
$$

holds for $\theta_{\mathfrak{W}}=19 \pi / 36=95^{\circ}$. In this expression, $\mathbf{x}$ is a point in $\mathcal{G}^{n+1}$, and $\hat{\mathbf{n}}$ and $\hat{\mathbf{u}}$ are the normal and velocity unit vectors interpolated from data at $t^{n}$. Intuitively, eq. (17) selects nodes lagging behind the motion of the new interface. Our goal is to protect enhanced level-set values in $\phi^{n+1}$ from indiscriminate redistancing on points on one side of $\Gamma^{n+1}$ at the current iteration. Then, we resort to the conventional semi-Lagrangian scheme to smooth out the moving front from the opposite side at $t^{n+2}$. In other words, improving the free boundary trajectory is not a task of the MLSemiLagrangian() function alone; it demands a delicate balance between machine learning correction, selective reinitialization, and pure numerical advection. In particular, we should enable Algorithm 1 whenever it is its turn in the alternating strategy, and the constraints described in Remark 1 are valid (i.e., $\Delta t=h, C F L=1$, and $\max \|\mathbf{x}\|=1$ for all $\mathbf{x} \in \Omega$ ). These conditions guarantee that the input information for $\mathscr{F}_{c, f}(\cdot)$ is compatible with the data collected during training. Furthermore, as shown in Section 5.4, alternating between solvers is necessary not only at the inference stage but also when optimizing our numerical-error estimator.

\subsection{Data-packet collection for learning and inference}

The CollectDataPackets() subroutine in Algorithm 2 is a straightforward procedure that exploits the multiprocess interpolation mechanism of [57] to extract information from local cells owning departure points. This information is necessary during inference (see Algorithm 1) and for constructing the training data set (see Section 5.4).

We begin Algorithm 2 by extracting a set $n$ of locally owned nodes near $\Gamma$. Every node $n \in n$ with coordinates $n . \mathbf{x}=(n . x, n . y)$ has an $h$-uniform, nine-point stencil and satisfies at least one of the four conditions: $\phi(n . x, n . y) \cdot \phi(n . x \pm$ $h, n . y) \leqslant 0$, or $\phi(n . x, n . y) \cdot \phi(n . x, n . y \pm h) \leqslant 0$. Figure 6 portrays a group of vertices typically found in $n$.

For each node $n \in \eta$, first, we compute its departure point $\mathbf{x}_{d}$ and midpoint velocity $\hat{\mathbf{u}}$ with eqs. (7) and (8) via quadratic interpolation. Next, we use $\mathbf{x}_{d}$ and $\hat{\mathbf{u}}$ to check the validity of $n$ and decide if we should continue processing it. A grid node is valid when its intermediate velocity is nonzero, and its departure point lies within a cell $C$ at the maximum level of refinement. Alongside the $C F L$ and velocity-norm restrictions, these new constraints help confine the problem's complexity and favor data consistency.

After validation, we fetch a partially populated $p$ using the information available for $n$ in $C$. To do so, we employ the FechDataPacket() subroutine, which extends the multi-process Interpolate() function. Upon calling 


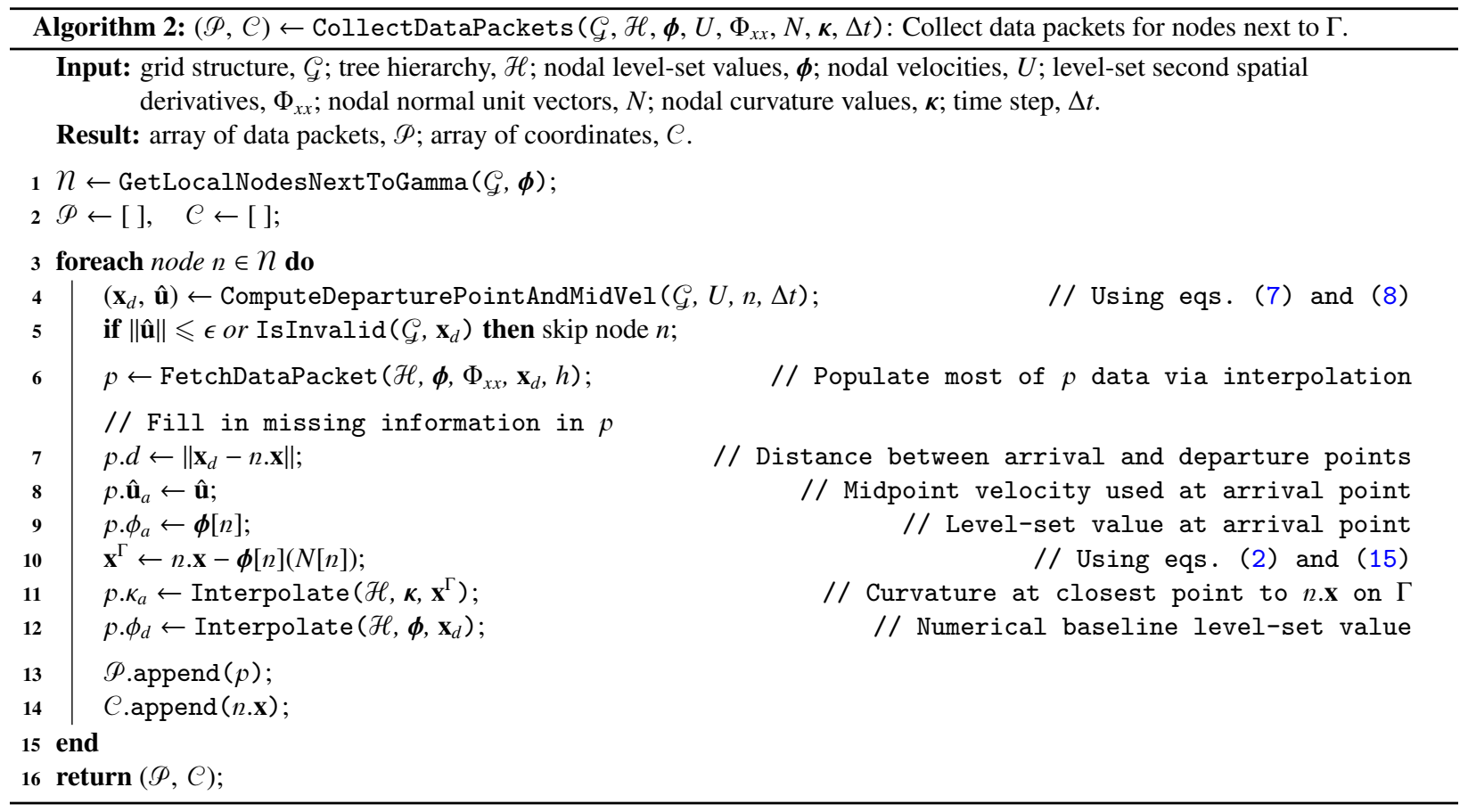

FechDataPacket ( $), p$ will hold data for $\left(\tilde{x}_{d}, \tilde{y}_{d}\right),(\phi)_{i j},\left(\mathbf{u}_{d}\right)_{i j},\left(\phi_{x x}\right)_{d}$, and $\left(\phi_{y y}\right)_{d}$. Then, we fill in the missing fields in $p$ by computing $n$ 's distance $d$ from $\mathbf{x}_{d}$ and querying its nodal level-set value $\phi_{a}$. In addition, we bilinearly interpolate $\kappa$ at $n$ 's projection onto $\Gamma$, as specified by eq. (15), and use quadratic interpolation to retrieve $\phi_{d}$ at $\mathbf{x}_{d}$, as given by eq. (7). Once more, note that our interpolative tasks require a local hierarchical reconstruction $\mathscr{H}$ to determine which partition owns $\mathbf{x}_{d}$. For a detailed description of the distributed interpolation procedure, we refer the reader to Algorithm 2 in [57]. By the end of our Algorithm 2, we will have all the valid-vertex data packets in $\mathscr{P}$ and their respective coordinates (i.e., arrival points) in $C$.

\subsection{Training}

The motivation for developing our error-correcting neural network stems from the promising results in machine learning applications that rest on image super-resolution methodologies [7, 13, 63-66]. Under these paradigms, to recover the information lost on a low-resolution grid, one should solve the same problem on a highly resolved mesh and assess the difference between both systems. In other words, the coarse-grid solution should mimic the fine-grid solution as much as possible with the help of the available data. In our case, we have already stated what information we can leverage when tracking the evolution of a moving front. Therefore, to train $\mathscr{F}_{c, f}(\cdot)$, we must assemble a data set $\mathscr{D}$ with curated low-resolution input data packets and high-resolution (departure) output target level-set values. Later, we can contrast the inferred $\phi_{d}^{\star}$ values in fig. 4 with their reference counterparts to optimize $\mathscr{F}_{c, f}(\cdot)$ via stochastic gradient descent.

\subsubsection{Data-set generation}

First, we explain how to build the learning data set $\mathscr{D}$. The high-level recipe is straightforward as long as we consider just one coarse resolution at a time. If various mesh sizes are necessary, one must produce their corresponding data sets separately and train their respective neural networks. This idea would be the semi-Lagrangian-scheme equivalence to the curvature neural-network dictionaries introduced in previous studies [45, 46]. Now, assuming that we discretize $\Omega$ with adaptive Cartesian grids, let $\ell_{c}^{\max }$ and $\ell_{f}^{\max }$ be the coarse- and fine-mesh maximum levels of refinement (refer to fig. 1). As stated in Section 5.1, we presume that $\ell_{c}^{\max }<\ell_{f}^{\max }$ and $h_{c}>h_{f}$. Thus, to populate $\mathscr{D}$, it suffices to set up a direct numerical simulation (DNS) with a predefined level-set function. Then, one must advect its interface simultaneously on a coarse and a fine grid with an external velocity field and collect samples periodically until some $t^{\text {end }}$. To accommodate the alternating mechanism described in Section 5.2 and illustrated in fig. 3, we note that data collection takes place every other iteration. Furthermore, we can promote pattern variation and generalization 
if we repeatedly perform these steps for random configurations. This randomization technique is the backbone of the GenerateDataSet () procedure presented in Algorithm 3.

Algorithm 3 is combinatorial. For each velocity field, we draw a level-set-function base parameter from a range of evenly distributed values. Then, we spawn the proper initial level-set configuration at $N_{C}$ different places within the computational domain. For simplicity, we use circular-interface level-set functions whose base parameters are their radii, and initial configurations are their interface centers. In our case, $N_{F}=7$ velocity fields and $N_{C}=4$ locations per radius yield well-sized data sets from simulations that last up to $t^{\text {end }}=0.5$. Also, to minimize concurrency issues, we carry out Algorithm 3 in a uni-process system that can fit a computational 2-by-2 discretized $\Omega \equiv[-1,+1]^{2}$ with four unit-square quadtrees.

As mentioned before, if $C F L=1$ and $\max \|\mathbf{u}(\mathbf{x})\|=1, \forall \mathbf{x} \in \Omega$, all nodes of interest will coincide with one vertex in their departure-point-owning cells (see fig. 5). By enforcing this constraint, we ensure that those departure points never lie farther than two cells away from $\Gamma^{n}$. Consequently, it is enough to require a coarse-grid uniform band around $\Gamma^{n}$ of half-width $B_{c}=2$ (measured in cell diagonals) in anticipation of Algorithm 2's validity test. For accuracy, we also impose a uniform band of half-width $B_{f}$ in the fine grid. While $B_{c}$ aids with data consistency and problem simplification, $B_{f}$ favors precision when querying target level-set values. In particular, $B_{f}$ is relevant to how we interleave advection of low- and high-resolution level-set values: first, we advance the fine-grid level-set function; then, we use that updated trajectory to determine which reference (i.e., more accurate) level-set values should become targets to the sampled coarse-grid arrival points. Therefore, if $\Delta t_{c}$ is the coarse time step, the advected-fine-mesh band around $\Gamma_{f}\left(t^{n}+\Delta t_{c}\right)$ must be wide enough to encompass the coarse-grid, selected nodes at time $t^{n}$. The expression for setting $B_{f}$ in the preamble of Algorithm 3 reassures these properties and a bit more. Since we have discretized $\Omega$ with unit-square Cartesian quadtrees, the ratio $h_{c}: h_{f}$ from coarse to fine mesh size is a power of two. Accordingly, after advecting the high-resolution level-set function, we find that the sampled coarse-grid points overlap with vertices within the uniform band around the new fine-grid zero-isocontour. The latter is a desirable feature when assembling $\mathscr{D}$, considering that we use interpolation to fetch information from the fine mesh.

Following the initialization of $h_{c}$ and $h_{f}$, we set the bounds for the interface radius. With these mesh sizes, we also define $N_{r}$, which is the number of radii to evaluate for every velocity field. Here, $N_{r}$ is equivalent to over three times as many coarse cells fit between $r_{\max }$ and $r_{\min }$. Then, we enter the outermost data production loop, where we repeatedly generate divergence-free, random velocity fields $V_{i}(\cdot)$, for $i=1,2, \ldots, N_{F}$. In this study, we have realized $V_{i}(\cdot)$ as a smooth field constructed from superpositions of sinusoidal waves [82], as implemented by [63] and [64]. Subsequently, we normalize these fields so that $\max \left\|\mathcal{V}_{i}(\mathbf{x})\right\|=1$, for all $\mathbf{x} \in \Omega$.

After defining $V_{i}(\cdot)$, we instantiate circular-interface level-set functions of the form

$$
\phi_{\circ}(\mathbf{x})=\left\|\mathbf{x}-\mathbf{x}_{\circ}\right\|^{2}-r_{\circ}^{2}
$$

with evenly spaced radii $r_{\circ}$ from $r_{\min }$ to $r_{\max }$. For each radius, we choose $N_{C}$ random centers $\mathbf{x}_{\circ}=\left(x_{\circ}, y_{\circ}\right)^{T}$ within the unit square $[-1 / 2,+1 / 2]^{2} \subset \Omega$. Then, we use the resulting $\phi_{\circ}(\mathbf{x})$ to create the starting coarse and fine grids, $\mathcal{L}_{c}^{0}$ and $\mathcal{L}_{f}^{0}$, with uniform bands around $\Gamma^{0}$. Next, we evaluate $\phi_{\circ}(\cdot)$ and $V_{i}(\cdot)$ on both meshes and conclude with preparatory reinitialization before interleaving advection and collecting samples. To improve the accuracy on the fine grid, we use $B_{c}$ times as many redistancing operations on the nodal level-set values $\boldsymbol{\phi}_{f}^{0}$ as we do on the coarse-grid $\boldsymbol{\phi}_{c}^{0}$. In this study, we have selected $v=10$ iterations for solving eq. (3), which is typical for many level-set applications. For example, Mirzadeh et al. opted for 20 steps in [57], but we have chosen half of it because eq. (18) is not far from a sign distance function, and we only need accurate results within a small band around $\Gamma$. In this regard, the practitioner could choose a larger $v$ to improve level-set smoothness but should keep in mind that redistancing is costly when one uses high-order schemes [83]. $v$ is thus another parameter limiting the scope of $\mathscr{F}_{c, f}(\cdot)$; the reason is that one must determine $v$ ahead of time depending on the accuracy-performance tradeoff. To conclude, Algorithm 3 launches the IntAdvectAndCollectSamples() procedure and accumulates the output samples into the learning data set $\mathscr{D}$.

We present the coarse- and fine-grid simultaneous advection subroutine to assemble $\mathscr{D}$ in Algorithm 4. Our method follows the natural flow of a DNS on $\mathscr{G}_{c}$ with interleaved updates to the level-set function on $\mathscr{G}_{f}$. In addition, it integrates the alternation depicted in fig. 3 and the periodic resetting operations necessary for trajectory synchronization. The formal parameters of Algorithm 4 include the initial-condition level-set vectors and velocity matrices. Also, it requires the divergence-free velocity function $V(\cdot)$ and the coarse- and fine-grid data structures. As before, our constraints help define the coarse-grid time step as $\Delta t_{c}=h_{c}$ (and $\Delta t_{f}=h_{f}$ ) since max $\|V(\mathbf{x})\|=1$ for all points in $\Omega$.

In each iteration, the main simulation loop in Algorithm 4 first advances the fine-grid level-set function from $\boldsymbol{\phi}_{f}^{n} \equiv \boldsymbol{\phi}_{f}\left(t_{c}^{n}\right)$ to $\boldsymbol{\phi}_{f}^{n+1} \equiv \boldsymbol{\phi}_{f}\left(t_{c}^{n+1}\right) \equiv \boldsymbol{\phi}_{f}\left(t_{c}^{n}+\Delta t_{c}\right)$. That way, we know which reference level-set values are expected for arrival points next to the interface in $\mathscr{C}_{c}^{n}$ during sampling. The AdvectFineGrid() subroutine retrieves the 


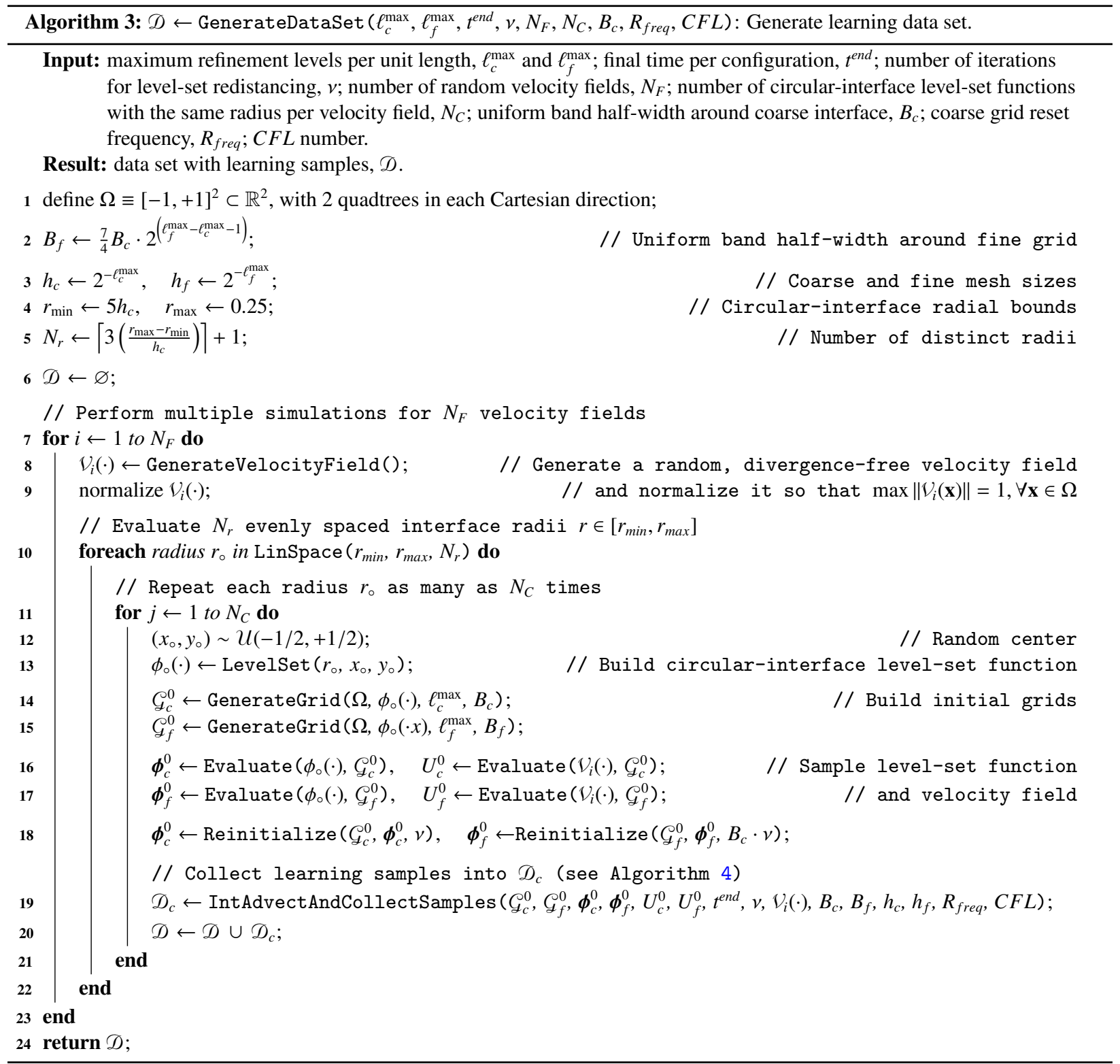

updated fine mesh and its nodal level-set values and velocities. Algorithm 5 describes the actions implied by this module. Briefly, Algorithm 5 is an iterative process based on the SemiLagrangian() scheme of [57]. It transports $\boldsymbol{\phi}_{f}^{n}$, reinitializes the new level-set values, and reevaluates $V(\cdot)$ on the updated grid at regular time intervals. About redistancing on $\mathscr{L}_{f}$, it is worth emphasizing that we need $B_{f} \cdot v$ iterations in the last fine-grid advection step. The motivation behind this idea is to ensure that $\phi_{f}^{n+1}$ accurately resembles a signed distance function as far as $B_{c}$ coarse cells away from $\Gamma_{f}$.

After advecting $\boldsymbol{\phi}_{f}^{n}$ to $\boldsymbol{\phi}_{f}^{n+1}$, we decide whether one should collect samples for the current iteration. If not, we simply call the AdvectCoarseGrid() module (see Algorithm 6) and retrieve the updated grid and (reinitialized) nodal level-set values into $\mathcal{L}_{c}^{n+1}$ and $\phi_{c}^{n+1}$. The AdvectCoarseGrid() subroutine is equivalent to a single step in Algorithm 5 performed on $\mathscr{G}_{c}$ with an additional reset operation. We will provide more details and the justification for the FitToFineGrid() reset statement below as we dive into data sampling.

In the case of a data-collection iteration, we start by computing coarse-grid nodal curvature values, normal unit vectors, and level-set second-order derivatives. Then, we fetch training input data through the CollectDataPackets () procedure listed in Algorithm 2. The sampling portion in IntAdvectAndCollectSamples() replicates the first 
Algorithm 4: $\mathscr{D}_{c} \leftarrow$ IntAdvectAndCollectSamples $\left(\mathcal{L}_{c}^{0}, \mathscr{L}_{f}^{0}, \phi_{c}^{0}, \phi_{f}^{0}, U_{c}^{0}, U_{f}^{0}, t^{\text {end }}, v, \mathcal{V}(\cdot), B_{c}, B_{f}, h_{c}, h_{f}, R_{f r e q}, C F L\right)$ : Interleave advection of coarse and fine grids and collect learning samples.

Input: initial coarse grid and its level-set values and velocities, $\mathscr{L}_{c}^{0}, \phi_{c}^{0}$, and $U_{c}^{0}$; initial fine grid and its nodal level-set values and velocities, $\mathscr{L}_{f}^{0}, \boldsymbol{\phi}_{f}^{0}$, and $U_{f}^{0}$; final advection time, $t^{e n d}$; number of iterations for level-set redistancing, $v$; velocity field, $V(\cdot)$; uniform band half-width around coarse interface, $B_{c}$; uniform band half-width around fine interface, $B_{f}$; coarse mesh size, $h_{c}$; fine mesh size, $h_{f}$; coarse-grid reset frequency, $R_{f r e q} ; C F L$ number.

Result: coarse-grid learning data set, $\mathscr{D}_{c}$.

$\mathbf{1} t_{c}^{n} \leftarrow 0, \quad \Delta t_{c} \leftarrow C F L \cdot h_{c} / \max \|\mathcal{V}(\mathbf{x})\|, \quad$ iter $\leftarrow 0 ;$

$\mathbf{2}\left(\mathcal{L}_{c}^{n}, \boldsymbol{\phi}_{c}^{n}, U_{c}^{n}\right) \leftarrow\left(\mathcal{L}_{c}^{0}, \boldsymbol{\phi}_{c}^{0}, U_{c}^{0}\right), \quad\left(\mathcal{L}_{f}^{n}, \boldsymbol{\phi}_{f}^{n}, U_{f}^{n}\right) \leftarrow\left(\mathcal{L}_{f}^{0}, \boldsymbol{\phi}_{f}^{0}, U_{f}^{0}\right) ;$

// Coarse-grid stepping variables

$3 \mathscr{D}_{c} \leftarrow \varnothing$

// Interleave advection of $\phi_{c}$ and $\phi_{f}$ using their respective structures in $\mathscr{G}_{c}$ and $\mathscr{G}_{f}$

4 while $t_{c}^{n}<t^{\text {end }}$ do

$5 \quad$ adjust $\Delta t_{c}$ if $t_{c}^{n}+\Delta t_{c}>t^{\text {end }}$;

$\left(\mathcal{L}_{f}^{n+1}, \boldsymbol{\phi}_{f}^{n+1}, U_{f}^{n+1}\right) \leftarrow$ AdvectFineGrid $\left(\mathcal{L}_{f}^{n}, \boldsymbol{\phi}_{f}^{n}, U_{f}^{n}, t_{c}^{n}, t_{c}^{n}+\Delta t_{c}, v, V(\cdot), B_{c}, B_{f}, h_{f}, C F L\right) ; \quad / /$ See Algorithm 5

// Alternating between sampling and standard advection

if 2 divides iter evenly then
$\left(\boldsymbol{\kappa}_{c}^{n}, N_{c}^{n}\right) \leftarrow$ ComputeCurvatureAndNormal $\left(\mathcal{L}_{c}^{n}, \boldsymbol{\phi}_{c}^{n}\right)$;
$\Phi_{c, x x}^{n} \leftarrow$ SecondSpatialDerivatives $\left(\mathscr{L}_{c}^{n}, \phi_{c}^{n}\right)$
// Nodal curvature and normal unit vectors
$\mathcal{H}_{f}^{n+1} \leftarrow \operatorname{Reconstruct}\left(\mathscr{L}_{f}^{n+1}\right), \quad \mathscr{H}_{c}^{n} \leftarrow \operatorname{Reconstruct}\left(\mathscr{L}_{c}^{n}\right)$;
$/ / \phi_{x x}$ and $\phi_{y y}$ at the nodes of $\mathcal{G}_{c}^{n}$
// Collect and process data packets to create learning samples
$(\mathscr{P}, \mathcal{C}) \leftarrow$ CollectDataPackets $\left(\mathcal{L}_{c}^{n}, \mathscr{H}_{c}^{n}, \boldsymbol{\phi}_{c}^{n}, U_{c}^{n}, \Phi_{c, x x}^{n}, N_{c}^{n}, \boldsymbol{\kappa}_{c}^{n}, \Delta t_{c}\right)$;
// See Algorithm 2
$m_{\phi} \leftarrow \varnothing$
// Map from node coordinates to $\phi$ values interpolated from $\phi_{f}^{n+1}$

foreach data packet $p \in \mathscr{P}$ do

let $\mathbf{x}_{a}$ be the coordinates (i.e., arrival point) corresponding to $p$ in $C$;

$\tilde{\phi}_{d}^{*} \leftarrow$ Interpolate $\left(\mathcal{H}_{f}^{n+1}, \boldsymbol{\phi}_{f}^{n+1}, \mathbf{x}_{a}\right) / h_{c} ; \quad / /$ Use advected fine grid to get normalized $\phi_{d}^{*}$

let $\boldsymbol{\xi}$ be the learning tuple $\left(p, \tilde{\phi}_{d}^{*}\right)$ with inputs $p$ and expected output $\tilde{\phi}_{d}^{*}$;

transform $\xi$, so that $p . \kappa_{a}$ is negative;

rotate $\xi$, so that the angle of $-p \cdot \hat{\mathbf{u}}_{a}$ lies between 0 and $\pi / 2$;

add tuple $\xi$ to $\mathscr{D}_{c}$;

let $\xi^{\prime}$ be the reflected tuple about line $y=x+\beta$ going through $\mathbf{x}_{a}$; add $\xi^{\prime}$ to $\mathscr{D}_{c}$;

// Data augmentation

$m_{\phi}\left[\mathbf{x}_{a}\right] \leftarrow h_{c} \tilde{\phi}_{d}^{*} ; \quad$ // Caching expected or improved level-set values

end

if $R_{\text {freq }}$ divides (iter +1$)$ evenly then

$\left(\mathscr{L}_{c}^{n+1}, \boldsymbol{\phi}_{c}^{n+1}\right) \leftarrow$ FitToFineGrid $\left(\mathscr{L}_{c}^{n}, \boldsymbol{\phi}_{c}^{n}, \mathscr{L}_{f}^{n+1}, \boldsymbol{\phi}_{f}^{n+1}\right) ; \quad$ // Reset coarse grid

else

$\left(\mathscr{L}_{c}^{n+1}, \boldsymbol{\phi}_{c}^{n+1}\right) \leftarrow$ SemiLagrangian $\left(\mathcal{L}_{c}^{n}, \boldsymbol{\phi}_{c}^{n}, U_{c}^{n}, U_{c}^{n}, C F L\right) ; \quad / /$ Refer to Algorithm 3 in [57]

AdjustLevelSetWithMLSolution $\left(\phi_{c}^{n+1}, m_{\phi}\right) ; \quad / /$ Correct $\phi^{n+1}$ for nodes with coords in $m_{\phi}$ end

W $\leftarrow$ GetCoordsWithNegativeFlow $\left(\mathcal{H}_{c}^{n}, U_{c}^{n}, N_{c}^{n}, \mathcal{L}_{c}^{n+1}, \boldsymbol{\phi}_{c}^{n+1}\right) ; \quad$ // Points 'lagging behind' $\Gamma_{c}^{n+1}$

$\boldsymbol{\phi}_{c}^{n+1} \leftarrow$ SelectiveReinitialization $\left(\mathcal{L}_{c}^{n+1}, \boldsymbol{\phi}_{c}^{n+1}, C \cap \mathscr{W}, v\right)$;

else

$\mid\left(\mathcal{L}_{c}^{n+1}, \boldsymbol{\phi}_{c}^{n+1}\right) \leftarrow \operatorname{AdvectCoarseGrid}\left(\mathcal{L}_{c}^{n}, \boldsymbol{\phi}_{c}^{n}, U_{c}^{n}, \mathcal{L}_{f}^{n+1}, \boldsymbol{\phi}_{f}^{n+1}, v\right.$, iter, $\left.R_{\text {freq }}, C F L\right) ; \quad$ // See Algorithm 6 end

$U_{c}^{n+1} \leftarrow \operatorname{Evaluate}\left(V(\cdot), \mathscr{L}_{c}^{n+1}\right)$

// Resample velocity field

iter $\leftarrow$ iter $+1, \quad t_{c}^{n} \leftarrow t_{c}^{n}+\Delta t_{c}$;

$\left(\mathcal{L}_{c}^{n}, \boldsymbol{\phi}_{c}^{n}, U_{c}^{n}\right) \leftarrow\left(\mathcal{L}_{c}^{n+1}, \boldsymbol{\phi}_{c}^{n+1}, U_{c}^{n+1}\right), \quad\left(\mathcal{L}_{f}^{n}, \boldsymbol{\phi}_{f}^{n}, U_{f}^{n}\right) \leftarrow\left(\mathcal{L}_{f}^{n+1}, \boldsymbol{\phi}_{f}^{n+1}, U_{f}^{n+1}\right) ;$

38 end

39 return $\mathscr{D}_{c}$; 


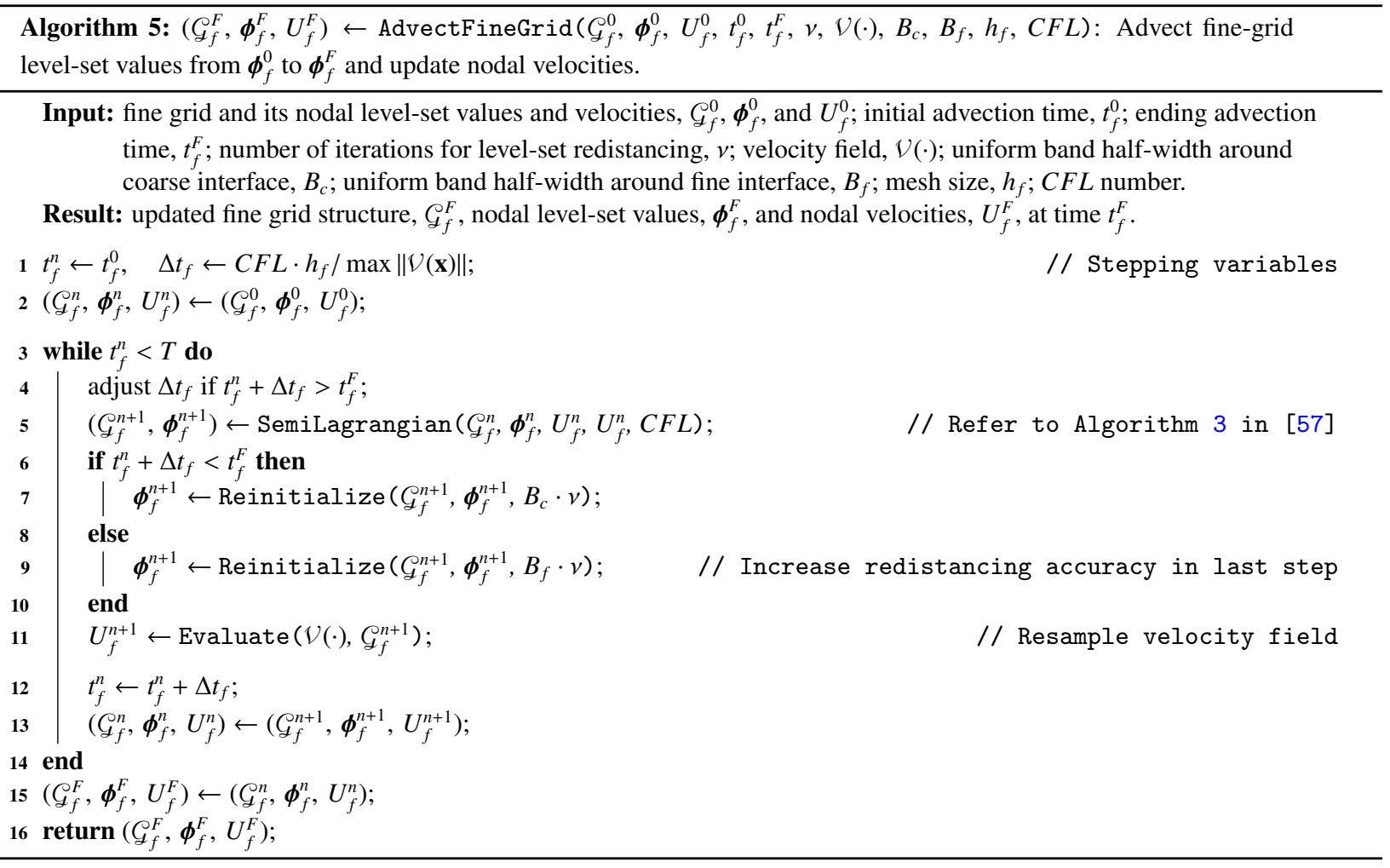

group of statements in our MLSemiLagrangian() algorithm. However, instead of constructing a batch of preprocessed entries, we accumulate learning tuples using the data packets in $\mathscr{P}$ as inputs. We denote a learning tuple by

$$
\xi \doteq\left(p, \tilde{\phi}_{d}^{*}\right) \in \mathbb{R}^{23}
$$

where $p$ appears in eq. (14), and $\tilde{\phi}_{d}^{*}$ is the $h_{c}$-normalized reference level-set value at the departure point. To calculate $\tilde{\phi}_{d}^{*}$, we turn to quadratic interpolation from $\phi_{f}^{n+1}$. This strategy leverages $\phi_{f}^{n+1}$, s higher accuracy and the fact that the coarse-grid vertices around $\Gamma_{c}^{n}$ are a proper subset of the fine-mesh uniform band around $\Gamma_{f}^{n+1} \equiv \Gamma_{f}\left(t^{n}+\Delta t_{c}\right)$. Similar to Algorithm 1, we reorient $p \in \xi$ into its standard form and apply negative-curvature normalization. At the same time, we store $\phi_{d}^{*}$ for next-to- $\Gamma_{c}^{n}$ nodes in a cache, $m_{\phi}$. Later, we will merge these target values with the rest of the elements in $\phi_{c}^{n+1}$ advected numerically.

In addition to accumulating reoriented-input samples, as in fig. $5 \mathrm{~b}$, we exploit symmetry and invariance in datapacket fields to supplement $\mathscr{D}$ with augmented pairs. Data augmentation via rotation and reflection has proven effective for image classifiers with convolutional networks $[1,3]$. Here, we augment samples by reflecting $p \in \xi$ about the line $y=x+\beta$ going through the origin of the local coordinate system centered at $p$ 's arrival point (see fig. 5c).

Next, we advance the coarse-grid level-set values after collecting learning samples for time $t^{n}$ into the cumulative set $\mathscr{D}_{c}$. To evolve $\phi_{c}^{n}$ to $\phi_{c}^{n+1}$ and update $\mathscr{L}_{c}^{n}$, we could have only called the SemiLagrangian() procedure and merged the reference $\phi_{d}^{*}$ values from $m_{\phi}$ back into $\phi_{c}^{n+1}$. However, the discretized coarse- and fine-mesh level-set functions can diverge to the point where our numerical-error characterization with $\mathscr{F}_{c, f}(\cdot)$ becomes invalid. Therefore, we reset $\boldsymbol{\phi}_{c}^{n+1}$ periodically through the FitToFineGrid() method by interpolating its level-set values from $\boldsymbol{\phi}_{f}^{n+1}$ every $\boldsymbol{R}_{\text {freq }}=3$ iterations. This recurrent synchronization protects against large trajectory deviations while preserving the natural noise that emerges from $\boldsymbol{\phi}_{c}^{n}$, s conventional advection.

The last steps in Algorithm 4's sampling iteration involve reinitializing $\boldsymbol{\phi}_{c}^{n+1}$. Such a task is subtle and requires special care in our machine learning strategy. In particular, we should solve eq. (3) only for values in $\boldsymbol{\phi}_{c}^{n+1}$ that we did not update via interpolation from $\phi_{f}^{n+1}$ and for which the condition in eq. (17) is not satisfied. Thus, one must use selective reinitialization to guard the machine-learning-corrected values with the help of the auxiliary arrays $C$ and $\mathcal{W}$. As shown in Section 6, the SelectiveReinitialization() and MLSemiLagrangian() subroutines work 


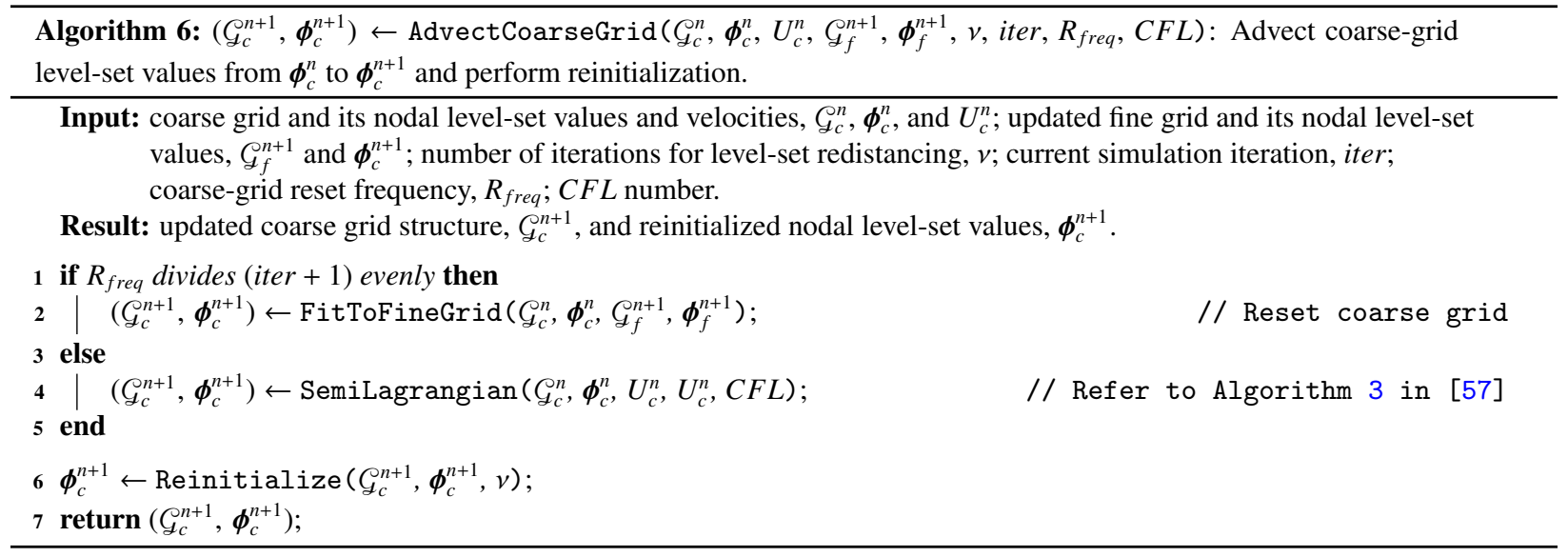

together with alternating transport to ensure that the moving front remains smooth and as close as possible to its higher-resolution counterpart.

The purpose of Algorithm 4 is to accumulate learning samples in a local set until we reach the final simulation time. Then, upon exiting IntAdvectAndCollectSamples (), we return to the GenerateDataSet () function, where $\mathscr{D}$ appends the partial results arriving from every DNS configuration. By the end of Algorithm 3 , $\mathscr{D}$ contains all the tuples in the form given by eq. (19). In the following tasks, we employ this data set to train and assess $\mathscr{F}_{c, f}(\cdot)$.

\subsubsection{Technical aspects}

Training an error-correcting neural network is much more resource-intensive than constructing its learning data set. Even though assembling $\mathscr{D}$ is algorithmically complex and can take several hours, one must perform this task just once at the beginning of the learning stage. On the contrary, optimizing $\mathscr{F}_{c, f}(\cdot)$ is less cumbersome but requires exploring the hyperparameter space through multiple evaluations. Every trial can last many hours depending on $|\mathscr{D}|$ and the size of the hidden layers in $\mathscr{F}_{c, f}(\cdot)$.

In this work, we have realized Algorithms 1 to 6 within our $\mathrm{C}++$ implementation of the parallel level-set framework of [57]. As for learning, we have simplified most of the tasks with TensorFlow [84] and Keras [85] in Python. In addition, we have followed conventional practices $[1,2]$ and split $\mathscr{D}$ into non-overlapping training, testing, and validation subsets to optimize $\mathscr{F}_{c, f}(\cdot)$ and promote generalization. Our partitioning approach also ensures that each subset mirrors the output $\tilde{\phi}_{d}^{*}$ distribution in $\mathscr{D}$. The specific heuristics that make this possible rely on the StratifiedKFold() method from Scikit-learn [86]. Although scientists usually reserve this balancing subroutine for classification problems, we have introduced it into our workflow by employing Pandas' cut() procedure [87]. The cut() function first allows us to bin the reference level-set values into discrete intervals. Then, we call StratifiedKFold() three times with $K=10$ to populate the training, testing, and validation subsets with $70 \%, 10 \%$, and $10 \%$ of the tuples in $D$. The remaining $10 \%$ is discarded as a way of performing balanced subsampling. However, we do not carry out the expensive full-fledged 10-fold cross-validation during hyperparameter tuning. We have observed that a single fold with a couple of random initializations is sufficient, given that $\mathscr{D}$ is large.

The other critical component in our machine-learning-extended semi-Lagrangian scheme is the Preprocess() module portrayed in figs. 3 and 4. The Preprocess () actions depend on training-subset statistics and help transform raw feature vectors into amenable representations for the numerical-error estimator [86]. In our hyperparameter-space exploration, we have discovered the existence of three types of preprocessing operations that favor convergence, interpretability, and precision. Algorithm 7 lists these adjustments performed on data-packet attributes before assembling the input vectors in eq. (16). First, we $h$-scale the distance-related data in $p$, such as the departure-arrival-point distance, level-set values, level-set second-order derivatives, and curvature. The purpose of $h$-scaling is (1) to reduce the relative difference among feature magnitudes in $p$ and (2) to uncorrelate data from a particular grid resolution. While (1) facilitates neural learning, (2) is useful for bootstrapping hyperparameter exploration across grid discretizations. In other words, uncorrelating data-packet attributes from the mesh size can reduce the cost of neural model optimization because the hyperparameters that work for $h_{c}$ are a good starting point for fitting $\mathscr{F}_{c, f}(\cdot)$ to learning tuples from $\mathscr{G}^{\prime}$, where $h_{c}^{\prime} \neq h_{c}$.

After $h$-scaling distance data, we use the feature-type-based statistics 2 extracted from the training subset to 


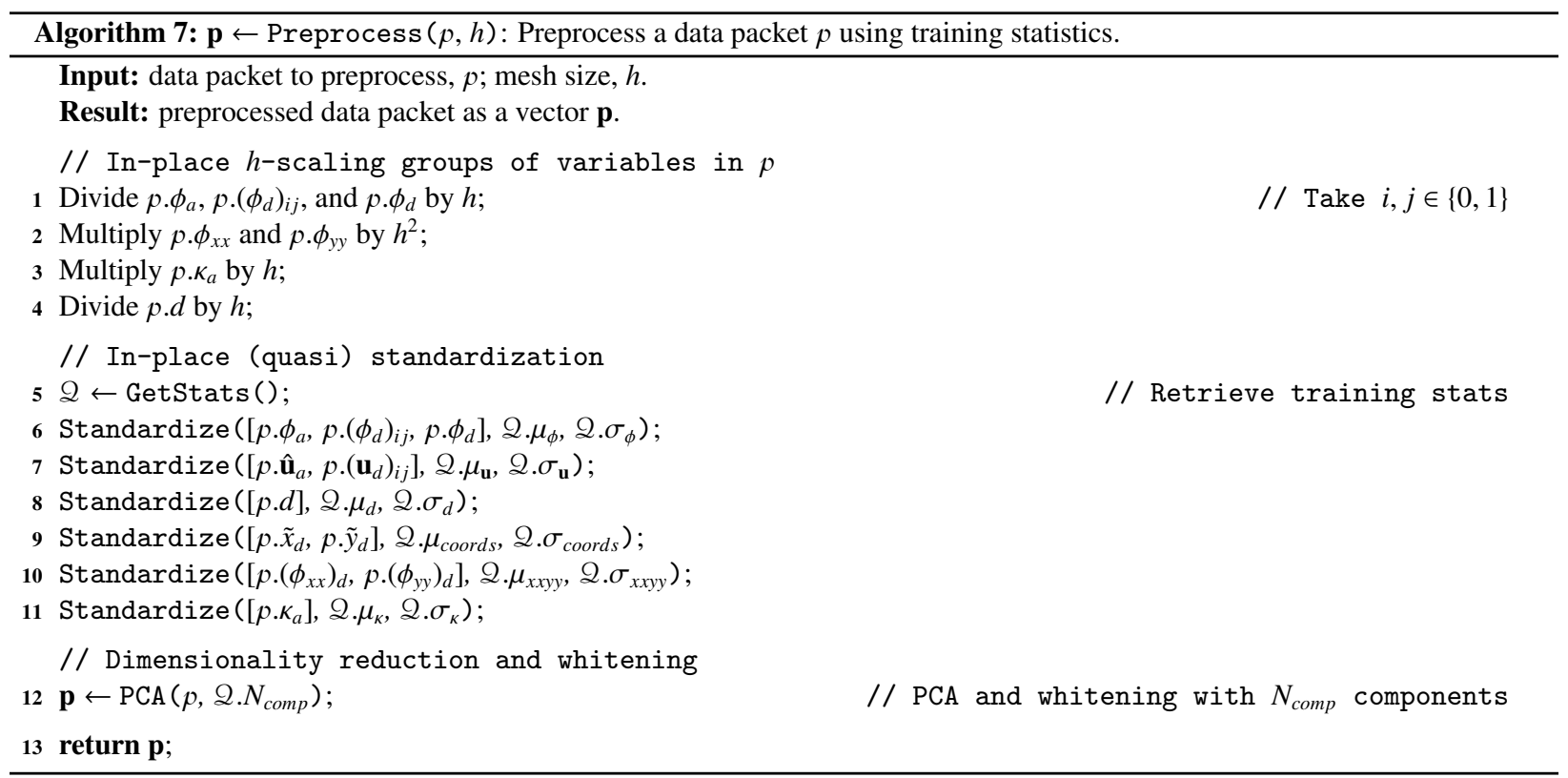

perform standardization. The latter comprises centering and scaling the entries in $p$ using the mean and standard deviation calculated across features of the same kind. For example, the Standardize() method in Algorithm 7 replaces $\varphi \in \Psi \subset p$ for $\left(\varphi-2 . \mu_{\phi}\right) / \mathcal{Q} . \sigma_{\phi}$, where 2. $\mu_{\phi}$ and $2 . \sigma_{\phi}$ are the level-set mean and standard deviation computed for $\Psi \doteq\left\{\phi_{a},\left(\phi_{d}\right)_{i j}, \phi_{d}\right\}$, where $i, j \in\{0,1\}$.

In the last group of preprocessing operations, we use principal component analysis (PCA) and whitening alongside dimensionality reduction. PCA and whitening are well known for accelerating convergence, and practitioners have shown that their combination leads to better results than z-scoring [46, 88]. Coupling standardization and PCA is also beneficial because it prevents variance from directly reflecting the scale of the data [80]. As for whitening, we have incorporated it because it adds a secondary component-wise scaling that raises uncorrelated concepts to the same level of importance (on an a priori basis) [1]. In our case, we employ Scikit-learn's PCA class to transform standardized data packets into input vectors with $2 . N_{c o m p}=17$ entries. After settling down with an optimal model, we export $\mathscr{F}_{c, f}(\cdot)$, Q, and the PCA object to JSON files. Then, we can use these files for porting the entire inference system in fig. 4 to different platforms.

We close this section by providing insight into training $\mathscr{F}_{c, f}(\cdot)$ with TensorFlow and Keras. As seen in fig. 4, our multilayer perceptron has $17+1$ linear input neurons that ingest data from the Preprocess() module and $\tilde{\phi}_{d}$. The first four hidden layers contain $N_{h}^{i}$ nonlinear, ReLU units, for $i=1,2,3,4$, with trainable connections and parameters. The last hidden-layer neuron is linear and updatable, and the output unit merely adds its input tensors while requiring no trainable weights. To train $\mathscr{F}_{c, f}(\cdot)$, we have used backpropagation [1, 89] with the Adam optimizer [90] to minimize the root mean squared error loss (RMSE) between $\tilde{\phi}_{d}^{*}$ and $\bar{\varepsilon}+\tilde{\phi}_{d}$. Likewise, we have introduced kernel regularization in all hidden layers with an L2 factor of $10^{-6}$. As for the weight updates, these take place after evaluating the RMSE over batches with 64 samples. In addition, we monitor the mean absolute error (MAE) on the validation subset to reduce the learning rate systematically and prevent overfitting. In particular, we stabilize optimization by attaching a callback function that halves the learning rate from $1.5 \times 10^{-4}$ to $1.5 \times 10^{-5}$ whenever the validation MAE does not improve for fifteen epochs. Also, we stop backpropagation as a protective measure if the latter MAE continues to deteriorate for fifty iterations. In our preliminary experiments, we have observed that a maximum of one thousand iterations suffices for training $\mathscr{F}_{c, f}(\cdot)$. Thanks to Keras' facilities and callback methods, we can easily handle these and other technical aspects, like best-model checkpoints and logging. The upcoming section asserts this complete process by producing an error-correcting neural network for a pair $(c, f)$ of grid resolutions.

\section{Results}

We now assess the feasibility of the ideas presented in Section 5. Our proof of concept comprises a neural model $\mathscr{F}_{6,8}(\cdot)$ designed for a coarse grid composed of unit-square quadtrees with $\ell_{c}^{\max }=6$. As for the fine grid that supplies 


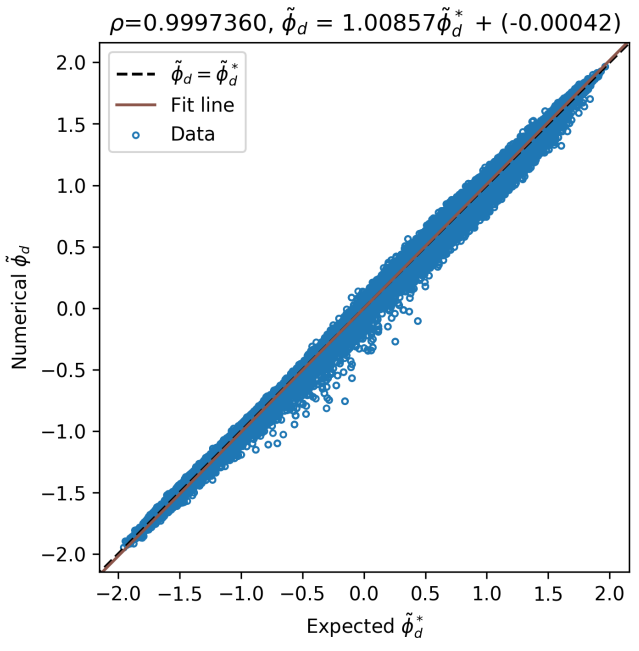

(a) Numerical semi-Lagrangian scheme

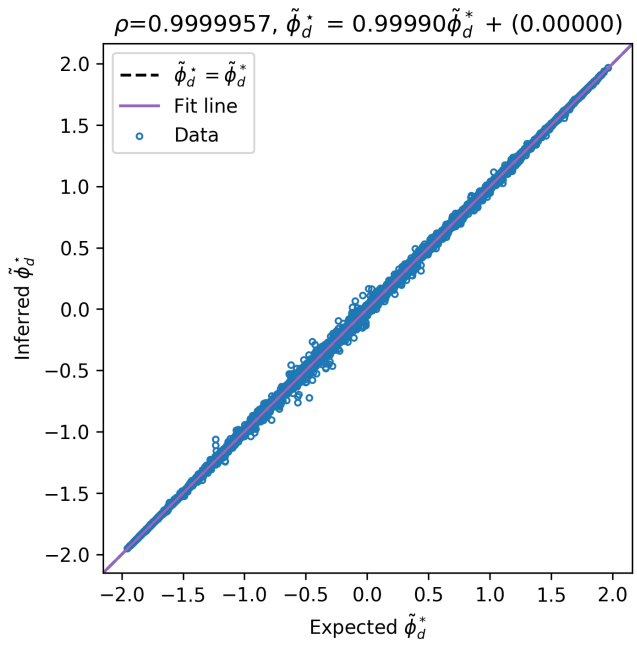

(b) Neural network

Figure 7: Learning correlation plots over $\mathscr{D}^{\prime}$ between expected and approximated $h_{c}$-normalized level-set values at the departure points. (Color online.)

the reference level-set values $\phi_{d}^{*}$, we require $\ell_{f}^{\max }=8$ and $h_{f}=2^{-8}$. Consequently, our error-correcting neural network, the MLSemiLagrangian() subroutine, selective reinitialization, and the SemiLagrangian() scheme must operate in tandem to improve interface advection with $h_{c}=2^{-6}=0.015625$.

To optimize $\mathscr{F}_{6,8}(\cdot)$, we gathered 4'051,946 samples from the methodologies outlined in Algorithms 2 to 6 . Then, we used stratified $K$-fold indexing (see Section 5.4.2) to discard about $10 \%$ of them and allocate $2^{\prime} 836,283,405,203$, and 405,213 tuples for the training, testing, and validation subsets. Hence, our curated learning set $\mathscr{D}^{\prime}$ ended up with 3'646,699 samples. Exploring the hyperparameter space with $\mathscr{D}^{\prime}$ resulted in an optimal architecture with $N_{h}^{i}=130$ units in the first four hidden layers. The topology in fig. 4 thus gave rise to 53,561 parameters that we trained during 337 epochs. At this stage, we also discovered that reducing input vectors from 22 to 17 dimensions was critical for learning stability and accuracy. While fewer than 17 components in the PCA object led to underfitting, more than these did not translate into any significant improvements. Likewise, employing over 17 dimensions precipitated outliers' appearance because of the additional whitening normalization. This problem is not new, and we have reported it when computing curvature in [46].

Figure 7 contrasts the fitting quality of the numerical baseline and $\mathscr{F}_{6,8}(\cdot)$ over the entire learning data set. These results confirm that the error-correcting function aids in narrowing the gap between the estimated and the reference level-set values during semi-Lagrangian advection. Table 1 supports this observation, where MaxAE corresponds to the maximum absolute error. Our neural network helps reduce the MAE by $91 \%$ and lowers the maximum error by a factor of 2.4 in $\mathscr{D}^{\prime}$. These figures corroborate that the conventional scheme in eqs. (6) to (8) can benefit from data-driven corrections even though we cannot represent $\bar{\varepsilon}$ exactly.

The following tests verify whether $\mathscr{F}_{6,8}(\cdot)$ can offset numerical dissipation and decrease artificial mass loss in physical simulations. These experiments include rotating and deforming a disk, measuring the effect of tangential shear flows, and solving two instances of the Stefan problem. For compatibility with $\mathscr{F}_{6,8}(\cdot)$, we have discretized $\Omega$ using quadtree Cartesian grids with unit-square macrocells and $\ell_{c}^{\max }=6$. Also, we ensure the simulations meet the conditions in fig. 5 by enforcing $C F L=1$ and scaling velocity fields to fit within the unit ball (i.e., $\Delta t=h_{c}$ ) whenever possible. For consistency, we reinitialize $\phi(\mathbf{x})$ every time step using ten iterations, as noted in Section 5.4.1. In all cases, we measure the level-set error norms defined by

$$
\ell^{1}=\frac{1}{n} \sum_{n \in n}\left|\phi(n . \mathbf{x})-\phi^{*}(n \cdot \mathbf{x})\right| \quad \text { and } \quad \ell^{\infty}=\max _{n \in n}\left|\phi(n . \mathbf{x})-\phi^{*}(n \cdot \mathbf{x})\right|,
$$

where $n$ is the set of nodes adjacent to $\Gamma$ (i.e., $|\phi(\mathbf{x})| \leqslant \sqrt{2} h_{c}$ ), and $\phi^{*}(\cdot)$ is the expected value. These collective statistics over a queried solution state are analogous to the MAE and MaxAE for $\phi_{d}$ introduced in the sections above.

We close this preamble with a remark about the hybrid inference system's technical implementation. In the follow- 


\begin{tabular}{|l|c|c|c|}
\hline & MAE & MaxAE & RMSE \\
\hline \hline Neural network & $7.990813 \times 10^{-4}$ & $2.535921 \times 10^{-1}$ & $1.744662 \times 10^{-3}$ \\
\hline Numerical semi-Lagrangian scheme & $9.113001 \times 10^{-3}$ & $6.033318 \times 10^{-1}$ & $1.471008 \times 10^{-2}$ \\
\hline
\end{tabular}

Table 1: Learning output statistics over $\mathscr{D}^{\prime}$ for $h_{c}$-normalized level-set values at the departure points.

\begin{tabular}{|l|c|c|c|c|r|r|}
\hline Method & $\ell_{c}^{\max }$ & $\ell^{1}$ error & $\ell^{\infty}$ error & Disk area & Area loss $(\%)$ & Time $(\mathrm{sec})$. \\
\hline \hline Ours & 6 & $4.672 \times 10^{-4}$ & $9.317 \times 10^{-4}$ & $7.027 \times 10^{-2}$ & 0.59 & 3.874 \\
\hline \multirow{3}{*}{ Numerical } & 6 & $3.380 \times 10^{-3}$ & $4.481 \times 10^{-3}$ & $6.740 \times 10^{-2}$ & 4.65 & 3.578 \\
& 7 & $8.545 \times 10^{-4}$ & $1.195 \times 10^{-3}$ & $6.985 \times 10^{-2}$ & 1.18 & 13.665 \\
& 8 & $2.152 \times 10^{-4}$ & $3.083 \times 10^{-4}$ & $7.048 \times 10^{-2}$ & 0.30 & 53.956 \\
\hline
\end{tabular}

Table 2: Rotation accuracy and performance assessment. Reported errors include measurements taken at grid points for which $\left|\phi_{\text {rot }}(\mathbf{x})\right| \leqslant \sqrt{2} h_{c}$ at the end of one revolution.

ing, we have turned to Lohmann's json suite [91] and some utilities from Hermann's frugally-deep library [92] to import $\mathscr{F}_{6,8}(\cdot)$ and the Preprocess () dependencies into $\mathrm{C}++$. frugally-deep provides a user-friendly interface for neural forward evaluations; however, we have reconstructed the internal mechanisms in $\mathscr{F}_{6,8}(\cdot)$ as a collection of weight matrices in OpenBLAS [93]. Compared with frugally-deep, OpenBLAS offers up to sixty times faster inference (with compiler optimization enabled ${ }^{7}$ ) but requires arranging inputs in a specific form. To accommodate OpenBLAS constraints, we clustered data into batches first. Then, we performed predictions in Algorithm 1 (line 14) with one 32-bit sgemm ${ }^{8}$ operation per simulation step. This lower-precision function has not affected our system's accuracy since $\mathscr{F}_{6,8}(\cdot)$ learned from $\mathscr{D}^{\prime}$ with the default single-floating-point TensorFlow configuration. Lastly, each of the coming tests has run in a computer with $16 \mathrm{~GB}$ RAM, $2.2 \mathrm{GHz}$ processor frequency, and no multi-threading. The reported wall times correspond to the shortest durations of ten repetitions for every experiment. We have made available $\mathscr{F}_{6,8}(\cdot)$ and related objects used in this section at https://github.com/UCSB-CASL/ECNet.

\subsection{Rotation}

Let $\phi_{\text {rot }}(\mathbf{x})$ be a starting level-set function, such as eq. (18), with a circular interface of radius $r=0.15$ centered at $(0,0.75)$ within $\Omega \equiv[-1,+1]^{2}$. If one considers the divergence-free velocity field

$$
\mathbf{u}_{r o t}(\mathbf{x})=\left(\begin{array}{c}
u(x, y) \\
v(x, y)
\end{array}\right)=\frac{1}{\sqrt{2}}\left(\begin{array}{c}
-y \\
x
\end{array}\right),
$$

the disk completes one revolution at $t^{\text {end }}=2 \pi \sqrt{2}$ since $\max \left\|\mathbf{u}_{r o t}(\mathbf{x})\right\|=1, \forall \mathbf{x} \in \Omega$. Table 2 shows our hybrid strategy's performance and the error for the interface location. In addition, it includes results for the baseline semi-Lagrangian scheme of [57] at various resolutions. Note that our approach comprises not only the MLSemiLagrangian() module but also a custom selective reinitialization. As interleaved advection in Algorithm 4, selective redistancing protects a subset of the machine-learning-corrected level-set values when solving eq. (3). Furthermore, we alternate between the numerical scheme and the MLSemiLagrangian() subroutine to regularize the inferred trajectory, as seen in fig. 3.

Compared with the baseline at $\ell_{c}^{\max }=6$, the statistics in table 2 prove that $\mathscr{F}_{6,8}(\cdot)$ helps counteract numerical diffusion. In particular, the average error reduces by a factor of 7.2, and the maximum error drops by around $79 \%$. Also important is the fact that artificial area loss decreases from $4.65 \%$ to $0.59 \%$. Further, table 2 reveals that our approach outperforms the numerical solver at twice the resolution. For instance, the hybrid system preserves area more accurately than the baseline at $\ell_{c}^{\max }=7$ but requires less than one-third of the computation time. For completeness, the last row of table 2 includes the results for the semi-Lagrangian scheme at the reference training resolution (i.e., $\ell_{c}^{\max }=8$ ). If one contrasts those measurements with ours, it is easy to see that the conventional method delivers smaller $\ell^{1}$ and $\ell^{\infty}$ error norms, as expected. This behavior echoes the findings of Zhuang et al. in [64], where an advecting neural model trained on a reference grid at $8 \times$ the resolution achieved roughly the same accuracy as the baseline at $4 \times$ the resolution. Extrapolating that rule into our work, we should anticipate area-preservation and level-set accuracy

\footnotetext{
${ }^{7}$ We used the flags $-02-03-$ march=native for $\mathrm{C}++14$.

${ }^{8}$ single-precision general matrix-matrix multiplication: $C=\alpha A B+\beta C$, where $\alpha=1$, and $\beta=0$ in our case.
} 

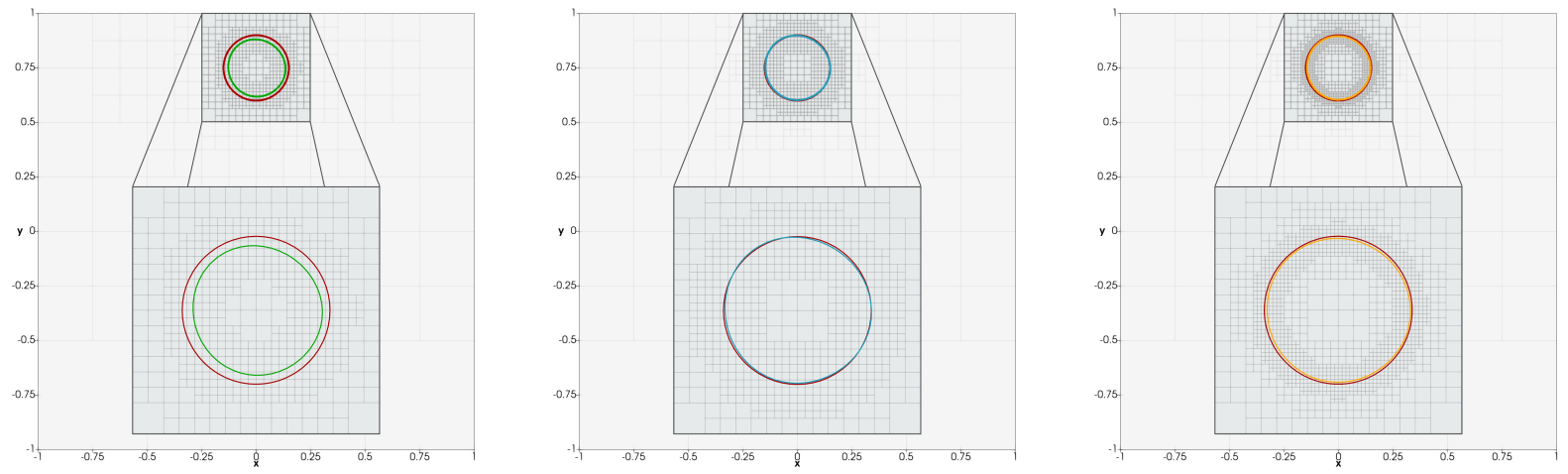

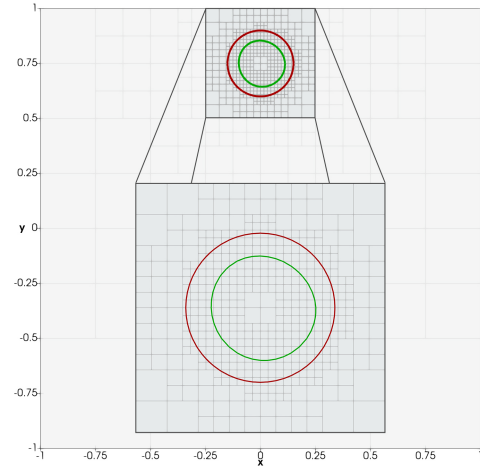

(a) Numerical baseline for $h_{c}=2^{-6}$

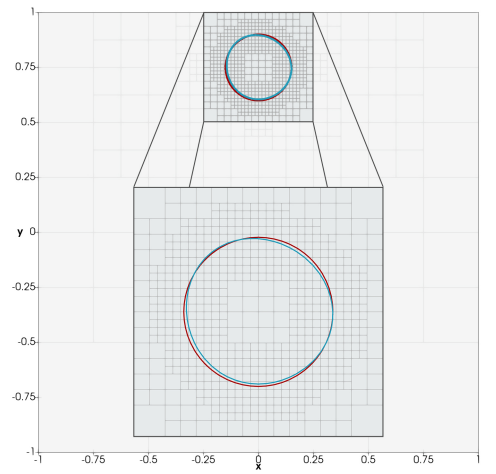

(b) Hybrid approach for $h_{c}=2^{-6}$

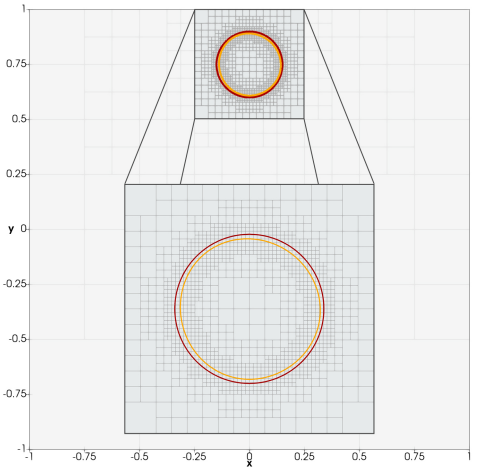

(c) Numerical scheme for $h_{c}=2^{-7}$

Figure 8: Rotation-problem zero level sets at the end of five (i.e., $t^{\text {end }}=10 \pi \sqrt{2}$ ) (top row) and ten (i.e., $\left.t^{\text {end }}=20 \pi \sqrt{2}\right)($ bottom row) revolutions. The left and right columns show the numerical scheme in green and orange for $\ell_{c}^{\max }=6$ and 7 . The center column provides the machine-learningcorrected solution in blue for $\ell_{c}^{\max }=6$. In all cases, the analytical disk appears in red. (Color online.)

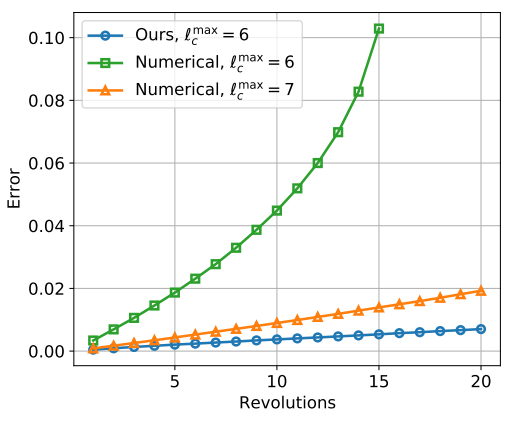

(a) Mean absolute error

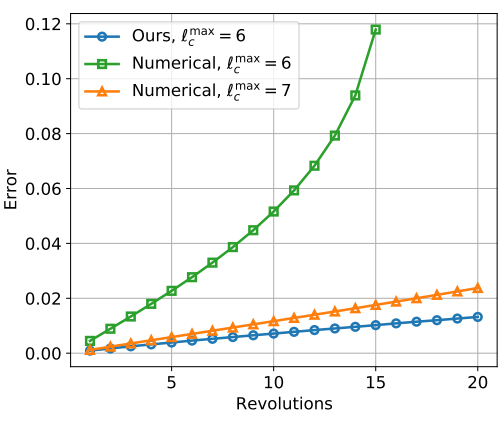

(b) Maximum absolute error

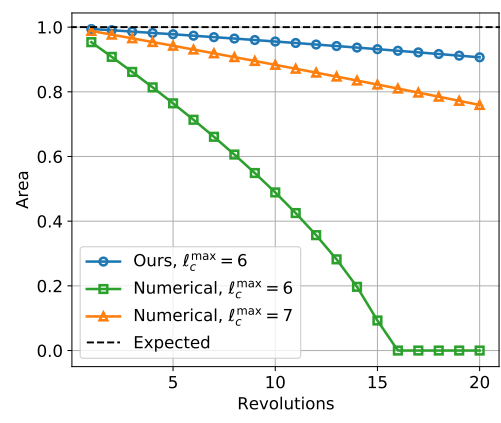

(c) Area conservation

Figure 9: Stress test for the rotation experiment using the semi-Lagrangian scheme and our hybrid advection system for $h_{c}=2^{-6}$. For comparison, we include the results for the numerical approach with $h_{c}=2^{-7}$. The left and center plots show the $\ell^{1}$ and $\ell^{\infty}$ error norms over $\phi$ at the end of each revolution. The right plot describes the evolution of the (normalized) area, taking $7.068583 \times 10^{-2}$ as a reference. (Color online.)

improvements analogous to the baseline outcomes at half the mesh size (i.e., $h_{c}=2^{-7}$ ). Figure 8 gives a visual perspective to our results corresponding to five and ten revolutions. We also include the standard solution for $\ell_{c}^{\max }=7$ to ease the comparison.

We conclude this experiment with a stress test. We compare the accuracy of our approach with the ordinary semiLagrangian scheme at the same $\left(\ell_{c}^{\max }=6\right)$ and twice the grid resolution $\left(\ell_{c}^{\max }=7\right)$. This time, the simulation lasts up to twenty revolutions. The resulting statistics appear in fig. 9. 


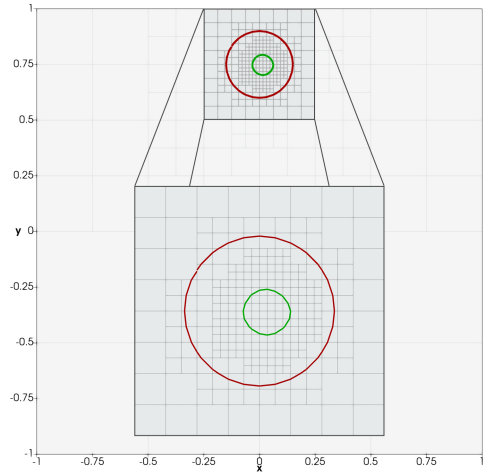

(a) Numerical baseline for $h_{c}=2^{-6}$

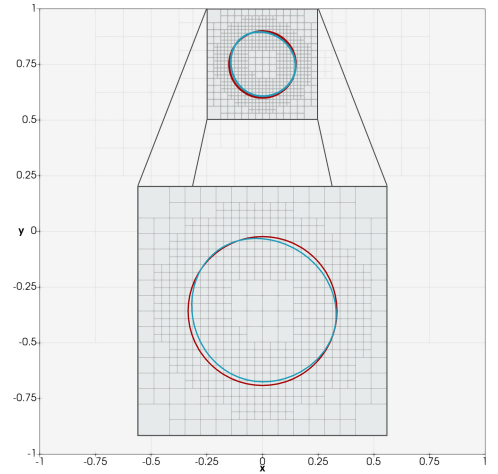

(b) Hybrid approach for $h_{c}=2^{-6}$

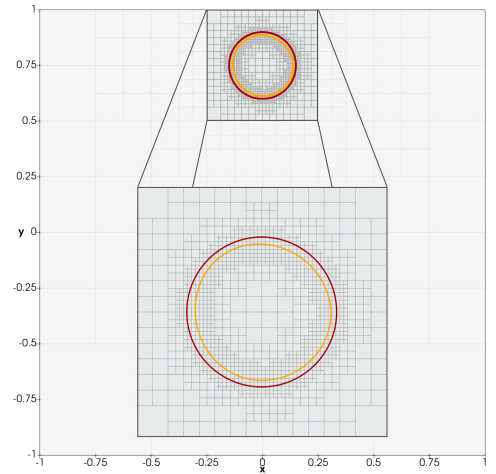

(c) Numerical scheme for $h_{c}=2^{-7}$

Figure 10: Rotation-problem zero level sets at the end of the fifteenth revolution (i.e., $t^{\text {end }}=30 \pi \sqrt{2}$ ). The left and center panels show the numerical baseline in green and the machine-learning-corrected solution in blue for $h_{c}=2^{-6}$. For comparison, we include the numerical results for a grid with twice the resolution (i.e., $h_{c}=2^{-7}$ ) in the right panel in orange. The analytical disk appears in red for all cases. (Color online.)

\begin{tabular}{|l|c|c|c|c|r|r|}
\hline Method & $\ell_{c}^{\max }$ & $\ell^{1}$ error & $\ell^{\infty}$ error & Disk area & Area loss $(\%)$ & Time $(\mathrm{sec})$. \\
\hline \hline Ours & 6 & $9.369 \times 10^{-4}$ & $3.751 \times 10^{-3}$ & $7.054 \times 10^{-2}$ & 0.20 & 0.656 \\
\hline \multirow{4}{*}{ Numerical } & 6 & $1.329 \times 10^{-3}$ & $5.686 \times 10^{-3}$ & $6.986 \times 10^{-2}$ & 1.17 & 0.602 \\
& 7 & $3.367 \times 10^{-4}$ & $2.361 \times 10^{-3}$ & $7.034 \times 10^{-2}$ & 0.49 & 2.369 \\
& 8 & $9.882 \times 10^{-5}$ & $9.487 \times 10^{-4}$ & $7.059 \times 10^{-2}$ & 0.14 & 9.715 \\
\hline
\end{tabular}

Table 3: Vortex-deformation accuracy and performance assessment. Reported errors include measurements taken at grid points for which $\left|\phi_{v t x}(\mathbf{x})\right| \leqslant$ $\sqrt{2} h_{c}$ at $t^{\text {end }}=1.25$.

Figure 9 confirms that the machine-learning-augmented semi-Lagrangian routine is superior to the baseline scheme at a compatible grid discretization with $h_{c}=2^{-6}$. Here, the corrected trajectory remains close to the analytical solution, whereas the numerical interface vanishes right after the fifteenth revolution. Our strategy also outperforms the numerical accuracy at twice the resolution. Specifically, $\mathscr{F}_{6,8}(\cdot)$ helped reduce area loss by a factor of 2.6 by the end of the twentieth rotation. The latter validates that our hybrid framework minimizes the effects of unwelcome diffusion on a comparable level with a conventional solver in a discretized domain with $h_{c}=2^{-7}$. Once more, these machine learning benefits come at a fraction of the cost required to solve the same problem in a mesh with eight-level unit-square quadtrees.

Figure 10 closes this case study with zero-isocontour illustrations at the end of the fifteenth revolution for the three tested systems. Finally, figs. 9 and 10 verify that our approach remains stable for all simulation times, independently from the chosen $t^{\text {end }}=0.5$ in Algorithm 3. However, as seen in fig. 10b, bias artifacts can emerge as $t^{n} \rightarrow \infty$. To tackle this phenomenon, we plan to enforce physical constraints, introduce temporal information during training, or consider a different numerical transport method.

\subsection{Vortex}

Next, we consider the more challenging vortex deformation flow proposed by [94]. Let $\phi_{v t x}(\mathbf{x})$ be an initial levelset function, such as eq. (18), with a circular interface of radius $r=0.15$ centered at $(0.5,0.75)$ within $\Omega \equiv[0,1]^{2}$. If we define the divergence-free velocity field

$$
\mathbf{u}_{v t x}(\mathbf{x})=\left(\begin{array}{c}
u(x, y) \\
v(x, y)
\end{array}\right)=\left(\begin{array}{c}
-\sin ^{2}(\pi x) \sin (2 \pi y) \\
\sin ^{2}(\pi y) \sin (2 \pi x)
\end{array}\right),
$$

the circle deforms into a (clockwise) spiral with $\max \left\|\mathbf{u}_{v t x}(\mathbf{x})\right\|=1, \forall \mathbf{x} \in \Omega$. In this experiment, we evolve the starting interface forward with eq. (22) until $t^{\text {mid }}=0.625$ and then backward using $-\mathbf{u}_{v t x}(\mathbf{x})$. We want to examine how accurately we can reconstruct the original disk at $t^{\text {end }}=1.25$.

Table 3 summarizes this experiment's interface-location accuracy and performance metrics for several discretizations. According to these statistics, our method is superior to the numerical baseline at $\ell_{c}^{\max }=6$, but it is not as good as 


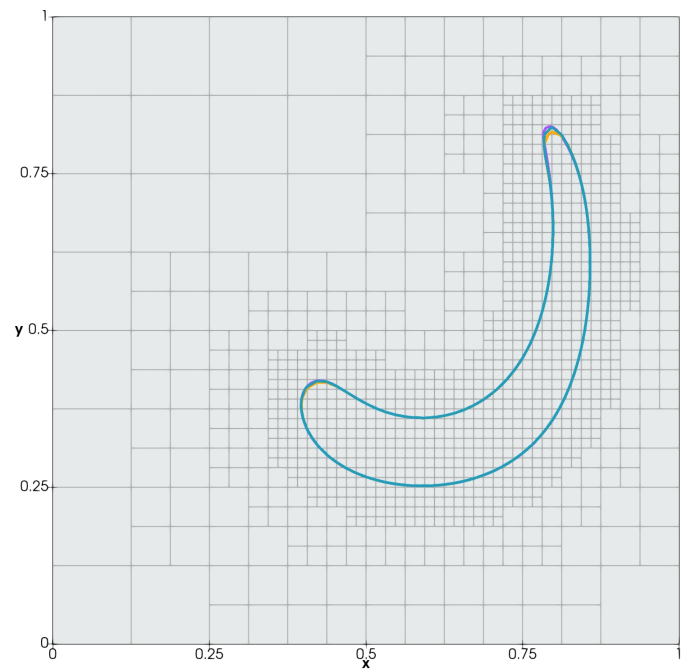

(a) Maximal deformation at time $t^{\text {mid }}=\frac{1}{2} t^{\text {end }}$

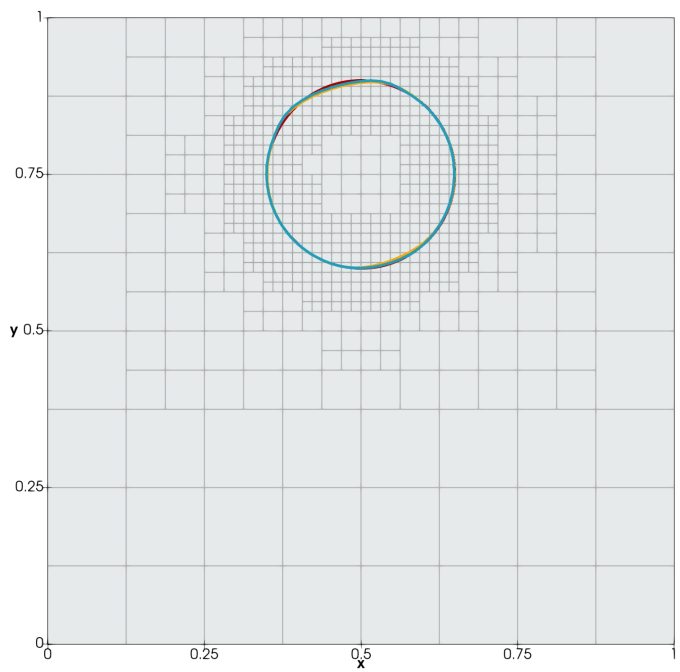

(b) Original disk reconstructed at time $t^{\text {end }}$

Figure 11: Zero level sets for the vortex experiment at the intermediate and final simulation times. The numerical baseline appears in orange for $\ell_{c}^{\max }=6$ and purple for $\ell_{c}^{\max }=7$. We show the machine-learning-corrected trajectory in blue for $\ell_{c}^{\max }=6$. For comparison, the analytical contour is only visible in the right panel in red. (Color online.)

\begin{tabular}{|l|c|c|c|r|}
\hline Method & $\ell^{1}$ error & $\ell^{\infty}$ error & Disk area & Area loss (\%) \\
\hline \hline Ours & $3.232 \times 10^{-3}$ & $1.164 \times 10^{-2}$ & $7.082 \times 10^{-2}$ & -0.20 \\
\hline Numerical & $4.185 \times 10^{-3}$ & $1.439 \times 10^{-2}$ & $7.021 \times 10^{-2}$ & 0.68 \\
\hline
\end{tabular}

Table 4: Degeneracy in the vortex-deformation experiment. Reported errors include measurements taken at grid points for which $\left|\phi_{v t x}(\mathbf{x})\right| \leqslant \sqrt{2} h_{c}$ at $t^{\text {end }}=2$ for $h_{c}=2^{-6}$.

the conventional semi-Lagrangian advection at twice the resolution (at least regarding the MAE). In particular, $\mathscr{F}_{6,8}(\cdot$ ) helps reduce the $\ell^{1}$ error norm by $30 \%$, the maximum error by one-third, and the area loss by $83 \%$ when $h_{c}=2^{-6}$.

Figure 11 confirms the findings in table 3 with visual evidence from the intermediate and final simulation states. Again, the machine learning solution requires a minimal fraction of the cost for realizing the numerical approach on finer grids. However, this time, it is more difficult for the proposed system to recover the accuracy lost to underresolution. For example, if the simulation prolongs to $t^{\text {end }}=2$, the hybrid strategy degenerates to being only slightly better than its numerical counterpart (see table 4). In this regard, we hypothesize that the observed behavior stems from the lack of physical constraints to enforce smoothness along the moving boundary. The mass gain in table 4 is probably a symptom of this problem, revealing our system's inability to redistribute area in highly deforming flows. Thus, this vortex test exposes the need to integrate more accurate smoothing mechanisms and improve the training protocol by encompassing more patterns from under-resolved regions.

\subsection{Circular-vortex-patch problem}

The following experiment measures the effects of tangential shear flows on a stationary interface. Let $\Gamma$ be the zeroisocontour of the level-set function $\phi_{c v p}(\mathbf{x})$ in eq. (18). Also, consider the simplified circular-vortex-patch problem, where a fixed disk $D_{c v p}$ of radius $r_{c v p}=0.6$, randomly centered at $\mathbf{x}_{c v p}=\left(x_{c v p}, y_{c v p}\right)^{T} \in\left[-h_{c} / 2,+h_{c} / 2\right]^{2}$, encloses a rotational velocity field given by

$$
\mathbf{u}_{c v p}(\mathbf{x})=\left(\begin{array}{ll}
u(x, y) \\
v(x, y)
\end{array}\right)= \begin{cases}\frac{1}{r_{c v p}}\left(\begin{array}{c}
-\left(y-y_{c v p}\right) \\
\left(x-x_{c v p}\right)
\end{array}\right), & \text { if }\left\|\mathbf{x}-\mathbf{x}_{c v p}\right\|<r_{c v p}, \\
\mathbf{0}, & \text { otherwise. }\end{cases}
$$

In other words, $D_{c v p} \subset \Omega \equiv[-1,1]^{2}$ contains a divergence-free velocity field, where $\left\|\mathbf{u}_{c v p}(\mathbf{x})\right\| \leqslant 1$ for all $\mathbf{x} \in D_{c v p}$, and $\mathbf{u}_{c v p}$ evaluates to zero everywhere else. Here, we assess how well we can preserve the initial circular profile 


\begin{tabular}{|l|c|c|c|c|r|r|}
\hline Method & $\ell_{c}^{\max }$ & $\ell^{1}$ error & $\ell^{\infty}$ error & Disk area & Area loss $(\%)$ & Time $(\mathrm{sec})$. \\
\hline \hline Ours & 6 & $6.839 \times 10^{-3}$ & $9.071 \times 10^{-3}$ & 1.105 & 2.28 & 5.207 \\
\hline \multirow{3}{*}{ Numerical } & 6 & $8.960 \times 10^{-4}$ & $1.239 \times 10^{-4}$ & 1.127 & 0.31 & 4.578 \\
& 7 & $5.709 \times 10^{-4}$ & $7.469 \times 10^{-4}$ & 1.129 & 0.19 & 19.035 \\
& 8 & $2.542 \times 10^{-4}$ & $3.523 \times 10^{-4}$ & 1.130 & 0.09 & 80.418 \\
\hline
\end{tabular}

Table 5: Circular-vortex-patch-problem accuracy and performance assessment. Reported errors include measurements taken at grid points for which $\left|\phi_{c v p}(\mathbf{x})\right| \leqslant \sqrt{2} h_{c}$ at $t^{e n d}=2 \pi r_{c v p}$.

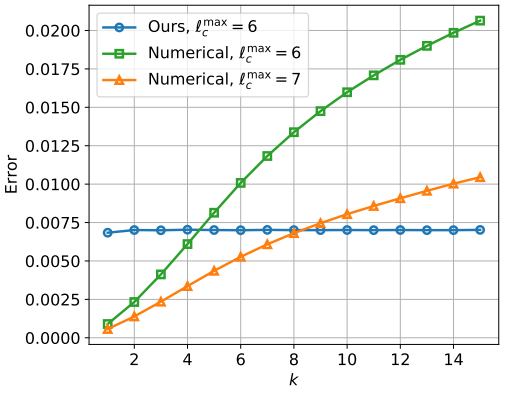

(a) Mean absolute error

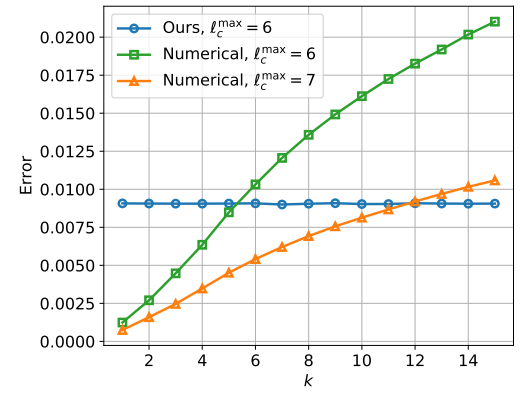

(b) Maximum absolute error

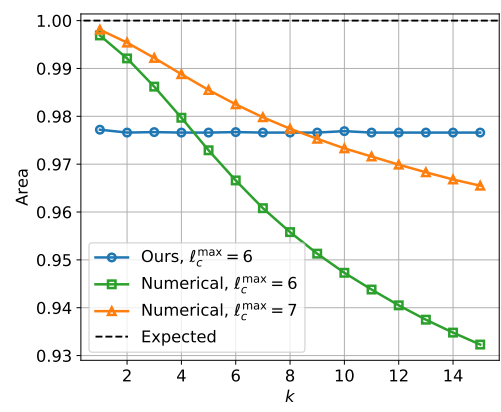

(c) Area conservation

Figure 12: Stress test for the circular-vortex-patch problem using the semi-Lagrangian scheme and our hybrid advection system for $h_{c}=2^{-6}$. For comparison, we include the results for the numerical approach with $h_{c}=2^{-7}$. The left and center plots show the $\ell^{1}$ and $\ell^{\infty}$ error norms over $\phi$ at the $k$ th revolution. The right plot describes the evolution of the (normalized) area, taking 1.130973 as a reference. (Color online.)

$\Gamma(0) \equiv D_{c v p}$ throughout a numerical simulation.

The error and performance statistics taken at $t^{\text {end }}=2 k \pi r_{c v p}$, for $k=1$, appear in table 5 for our system and the SemiLagrangian () method at three resolutions. $t^{\text {end }}$ is the time it would take for a particle to complete $k$ revolutions inside $D_{c v p}$, but $\Gamma(t)$ should not deform for any $t \geqslant 0$. As seen in table 5, our error-correcting neural network cannot produce satisfactory results for this short period. When $\ell_{c}^{\max }=6$, for example, our system cannot generalize to this kind of velocity data and worsens the trajectory quality by a factor of 7.3 in the $\ell^{\infty}$ error norm. However, table 5 does not tell the whole story, as we illustrate next.

Suppose we stress the baseline and the hybrid solvers with $k=15$ revolutions so that $t^{\text {end }}=30 \pi r_{c v p}$. If we track the boundary evolution and the error norms in eq. (20), we get the charts shown in fig. 12. Figure 13 supplements these results by contouring $\Gamma$ and $D_{c v p}$ at the end of the fifteenth rotation. Clearly, our strategy outperforms the baseline in the long run, even at twice the grid resolution. This phenomenon has been reported in [64], where neurally computed transport profiles gradually reached a steady state and then maintained those shapes forever. In our case, the alternating mechanism discussed above seems to lock the interface in equilibrium with the velocity field, which keeps $\Gamma$ 's area stable and independent of time. Although the blue circle in fig. 13b is not as regular as the orange disk in fig. 13c, our MLSemiLagrangian() subroutine can reduce advection costs by a factor of 3.65 , as seen in table 5 .

Our findings in this experiment expose a certain degree of overfitting in our error-correcting strategy. In the beginning, $\mathscr{F}_{c, f}(\cdot)$ cannot recognize the tangential shear-flow patterns. Thus, the machine-learning-corrected trajectory's precision declines fast as $\Gamma$ undergoes mild deformations. These deformations and velocity patterns eventually match $\mathscr{F}_{c, f}(\cdot)$ 's training tuples. Then, the mass-conservation scheme kicks in to preserve the blob's area indefinitely. In a sense, our neural network transforms the disk into a profile it knows how to handle. Therefore, if we must improve MLSemiLagrangian()'s robustness, we should start by integrating more velocity patterns and interface types into the training process. Also, we should make our method consistent by adding detection mechanisms to fall back to the numerical approximation whenever the latter is sufficiently accurate. This task might involve investigating neural classifiers (e.g., [29-31]) or some gradient-dependent quality metric (e.g., [95-97]) to recognize well-resolved stencils.

\subsection{Stefan problem}

We now assess the performance of our hybrid algorithm when solving the phase transition of a single-component liquid melt to a solid structure. Here, we consider a diffusion-dominated crystallization process that we can model as a Stefan problem. To state this FBP, suppose we split the computational domain $\Omega$ into two subdomains, $\Omega_{s}$ and $\Omega_{l}$, 


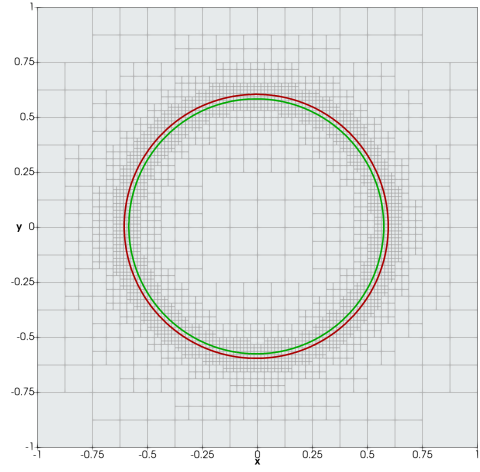

(a) Numerical baseline for $h_{c}=2^{-6}$

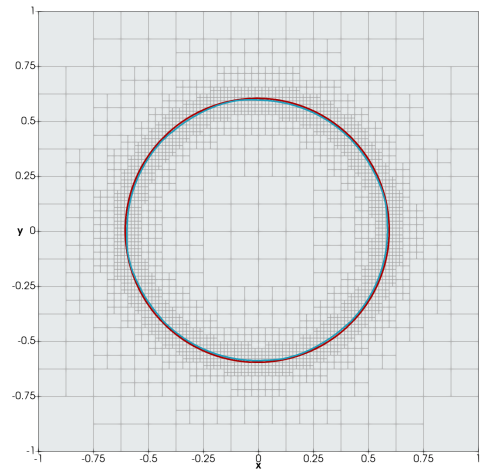

(b) Hybrid system for $h_{c}=2^{-6}$

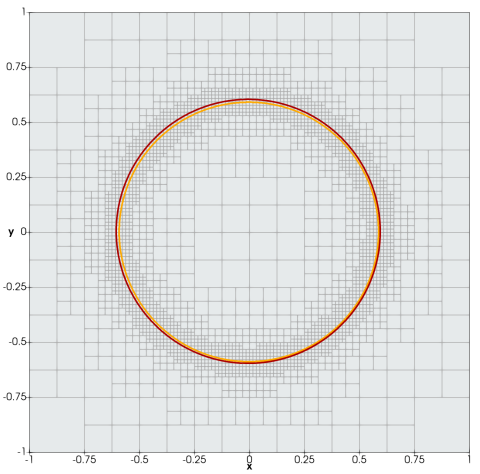

(c) Numerical solution for $h_{c}=2^{-7}$

Figure 13: Circular-vortex-patch zero level sets at $t^{\text {end }}=30 \pi r_{c v p}$. The baseline appears in the left and right panels in green and orange for $\ell_{c}^{\max }=6$, and 7. Our solution is shown in the center panel in blue. We display the analytical contour (i.e., $D_{c v p}$ ) in all charts in red. (Color online.)

separated by a solidification front $\Gamma$. The Stefan problem describes the evolution of the scalar temperature field $T$, decomposed into $T_{s}$ for the solid phase $\Omega_{s}$ and $T_{l}$ for the liquid phase $\Omega_{l}$, as ${ }^{9}$

$$
\begin{cases}\frac{\partial T_{l}}{\partial t}=D_{l} \Delta T_{l}, & \text { in } \Omega_{l}, \\ \frac{\partial T_{s}}{\partial t}=D_{s} \Delta T_{s}, & \text { in } \Omega_{s} .\end{cases}
$$

Generally, the $D_{l}$ and $D_{s}$ diffusion constants can be discontinuous across $\Gamma$. Moreover, the temperature on the solidliquid interface satisfies the Gibbs-Thomson boundary condition $[98,99]$

$$
T_{s}=T_{l}=T_{\Gamma}=-\epsilon_{c} \kappa-\epsilon_{v}\left(\mathbf{u}_{s p} \cdot \mathbf{n}\right) .
$$

In this expression, $\mathbf{n}$ is the outward unit vector normal to the solidification front, $\mathbf{u}_{s p}$ denotes the interface velocity, $\kappa$ is the local curvature at the interface, and $\epsilon_{c}$ and $\epsilon_{v}$ are the surface tension and molecular kinematics supercooling coefficients. As for the normal velocity component, we can compute it in terms of the jump in the heat flux at $\Gamma$ with

$$
\mathbf{u}_{s p} \cdot \mathbf{n}=-\left[\nabla_{\mathbf{n}} T\right]=-\left(D_{l} \nabla_{\mathbf{n}} T_{l}-D_{s} \nabla_{\mathbf{n}} T_{s}\right),
$$

where $\nabla_{\mathbf{n}} T$ is the directional derivative $\mathbf{n} \cdot \nabla T$. For the practical implementation of the Stefan problem in the parallel level-set framework, we refer the reader to Algorithm 5 in [57]. The latter extends the adaptive-Cartesian-grid-based Algorithm 2 in [55]. In addition, we have assumed $D_{s}=D_{l}=1$ and used PETSc's BiCGStab KSPSolve function [81] to solve the system arising from eq. (24).

\subsubsection{Frank-sphere}

First, we evaluate our system's response to the Frank-sphere problem [100], which has a known analytical solution. In this FBP, a region described by a disk with $T_{s}=0$ grows into a supercooled liquid phase. As time progresses, the radius expands according to $R(t)=s_{0} \sqrt{t}$, where $s_{0}$ is the base parameter. Likewise, we define the temperature field by

$$
T(r, t)=T(s)=\left\{\begin{array}{ll}
0 & \text { if } s \leqslant s_{0} \\
T_{\infty}\left(1-\frac{F(s)}{F\left(s_{0}\right)}\right) & \text { if } s>s_{0}
\end{array},\right.
$$

where $r$ is the distance to the disk's center, $s=r / \sqrt{t}$, and $F(s)=E_{1}\left(s^{2} / 4\right)$. Further, $E_{1}(z)=\int_{z}^{\infty} \frac{\mathrm{e}^{-t}}{t} \mathrm{~d} t$ is the exponential integral, and $\mathrm{F}(\mathrm{s})$ is the heat equation's similarity solution. Also, $T_{\infty}$ denotes the temperature infinitely far away from $\Gamma$. To determine $T_{\infty}$, we combine the jump condition in eq. (26) with the relation

\footnotetext{
${ }^{9}$ The subscripts $s$ and $l$ indicate the solid and liquid phases.
} 


\begin{tabular}{|l|c|c|c|c|c|r|r|}
\hline Method & $\ell_{c}^{\max }$ & $\ell^{1}$ error & $\ell^{\infty}$ error & Temperature error & Disk area & Area loss $(\%)$ & Time (sec.) \\
\hline \hline Ours & 6 & $5.853 \times 10^{-4}$ & $1.762 \times 10^{-3}$ & $4.225 \times 10^{-4}$ & $6.876 \times 10^{-1}$ & -0.05 & 4.342 \\
\hline \multirow{4}{*}{ Numerical } & 6 & $8.112 \times 10^{-4}$ & $3.625 \times 10^{-3}$ & $6.982 \times 10^{-4}$ & $6.892 \times 10^{-1}$ & -0.29 & 3.497 \\
& 7 & $9.253 \times 10^{-3}$ & $5.614 \times 10^{-2}$ & $9.137 \times 10^{-3}$ & $6.846 \times 10^{-1}$ & 0.38 & 9.253 \\
& 8 & $5.039 \times 10^{-2}$ & $1.511 \times 10^{-1}$ & $3.405 \times 10^{-2}$ & $6.879 \times 10^{-1}$ & -0.10 & 92.190 \\
\hline
\end{tabular}

Table 6: Frank-sphere accuracy and performance statistics. Reported errors include measurements taken at grid points for which $\left|\phi_{v t x}(\mathbf{x})\right| \leqslant \sqrt{2} h_{c}$ at $t^{\text {end }}=0.875$.

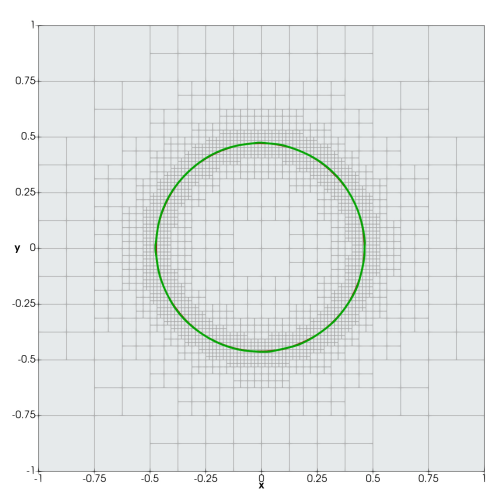

(a) Numerical baseline for $h_{c}=2^{-6}$

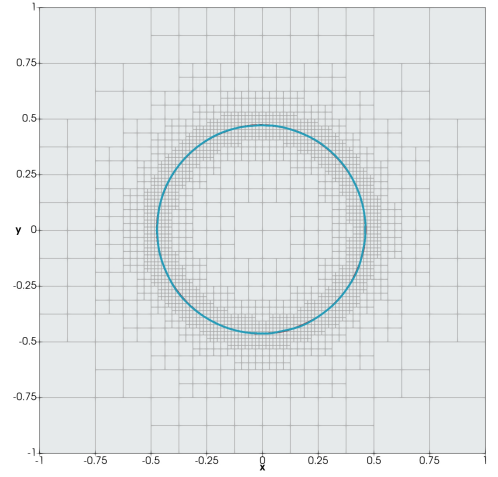

(b) Hybrid approach for $h_{c}=2^{-6}$

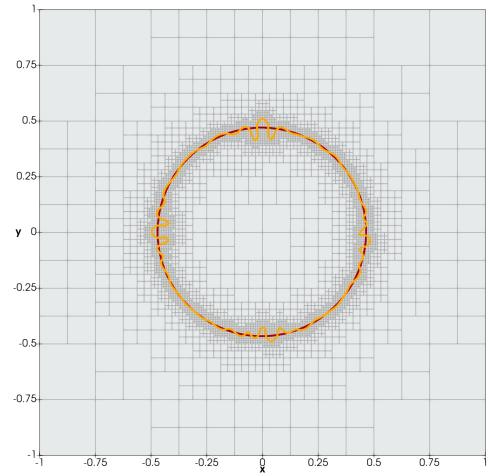

(c) Numerical scheme for $h_{c}=2^{-7}$

Figure 14: Frank-sphere zero level sets at $t^{\text {end }}=0.875$. The left and center panels show the numerical baseline in green and the machine-learningcorrected solution in blue for a discretization with $\ell_{c}^{\max }=6$. For comparison, we include the numerical result for a grid with twice the resolution in the right panel in orange. In all cases, the analytical disk appears in red. (Color online.)

$$
\mathbf{u}_{s p} \cdot \mathbf{n}=\frac{\mathrm{d} R}{\mathrm{~d} t}=\frac{s_{0}}{2 \sqrt{t}}
$$

at the freezing front so that

$$
T_{\infty}=\frac{s_{0} F\left(s_{0}\right)}{2 F^{\prime}\left(s_{0}\right)}
$$

In this experiment, $\phi_{f s}(\mathbf{x})=\left\|\mathbf{x}-\mathbf{x}_{f s}\right\|-r_{f s}$ is our initial level-set function embedded in $\Omega \equiv[-1,+1]^{2}$ with a circular interface centered at a random location $\mathbf{x}_{f s}$ inside $\left[-h_{c} / 2,+h_{c} / 2\right]^{2}$. If we define $s_{0}=0.5$ and $t^{0}=0.25$, we get $T_{\infty}=-0.15015425523242384$, and the freezing front radius $r_{f s}$ starts at 0.25 . With these parameters, we ensure that $\max \left\|\mathbf{u}_{s p}(\mathbf{x})\right\| \leqslant 1$ for all $\mathbf{x} \in \Omega$ upon quadratic extension from $\Gamma$. In addition, we enforce the time-step restriction $\Delta t=h_{c}$ and the Dirichlet boundary condition $T_{\Gamma}=0$.

Table 6 summarizes the interface-location and temperature error statistics for Frank-sphere simulations running until $t^{\text {end }}=0.875$. The table also shows the area loss and the running wall time at resolutions varying from $\ell_{c}^{\max }=6$ to $\ell_{c}^{\max }=8$. Because of the inherent instability in the Frank-sphere problem, the free boundary develops dendritic structures as the mesh size decreases. However, for $h_{c}=2^{-6}$, our strategy agrees with the baseline trajectory while exhibiting better area conservation and temperature accuracy. Figure 14 supplements these observations by contrasting the zero-isocontours computed with the numerical solver and the hybrid system. This test certifies the feasibility of our approach for building solutions for FBPs where the velocity field is not necessarily divergence-free.

\subsubsection{Unstable solidification}

At last, we evaluate our approach's response to curvature-driven, anisotropic crystallization. To this end, we place a solid seed into a supercooled liquid and solve eq. (24) with a nonzero Gibbs-Thomson condition (i.e., $T_{\Gamma} \neq 0$ ). Here, we consider the domain $\Omega \equiv[-1,+1]^{2}$ with a temperature field initialized uniformly as $T_{l}=-0.08$ for the liquid phase and $T_{s}=0$ for the seed. The nucleus has a characteristic three-fold freezing front given by the polar-rose equation 


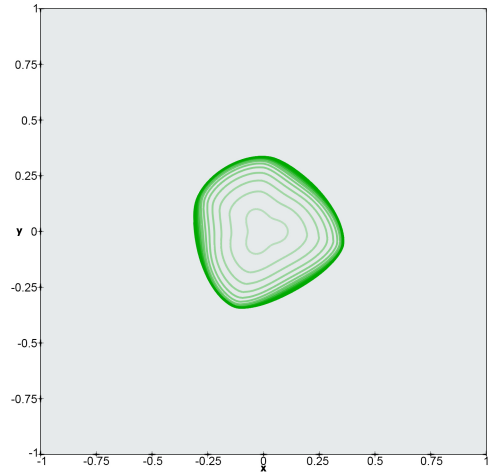

(a) Numerical baseline for $h_{c}=2^{-6}$

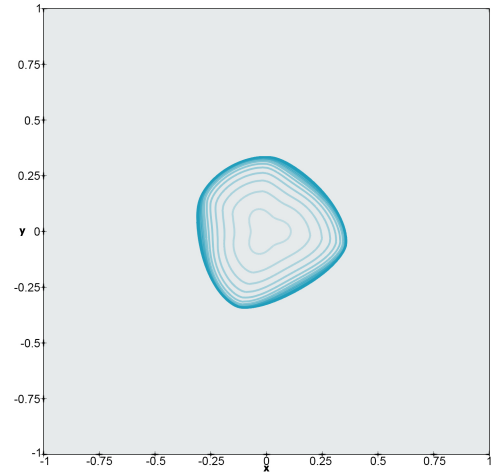

(b) Hybrid approach for $h_{c}=2^{-6}$

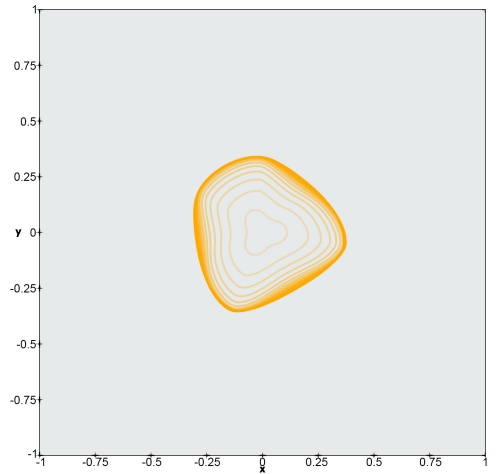

(c) Numerical scheme for $h_{c}=2^{-7}$

Figure 15: Unstable-solidification zero-level-set growth histories with sixteen steps from $t^{0}=0$ to $t^{\text {end }}=3$ for $\ell_{c}^{\max }=6,7$. The (green and orange) numerical trajectories appear on the left and right panels. We show our hybrid approach solution in the center chart in blue. (Color online.)

$$
r(\theta)=a \cos (p \theta)+b,
$$

where $a=0.02, b=0.09, p=3$, and $\theta \in[0,2 \pi)$. Furthermore, we have applied the condition in eq. (25) using

$$
\epsilon_{c}=2 \times 10^{-4}\left[1+0.6 \cos \left(4\left(\theta_{\mathbf{n}}+\frac{\pi}{3}\right)\right)\right] \text { and } \epsilon_{v}=0,
$$

where $\theta_{\mathbf{n}}$ is the interface normal-vector angle with respect to the horizontal. Also, we have imposed adiabatic boundary conditions for the four sides of $\Omega$. With this configuration, we have carried out the unstable-solidification simulation from $t^{0}=0$ to $t^{\text {end }}=3$.

Figures 15 and 16 compare the numerical and machine-learning-corrected growth histories and final zero level sets for $h_{c}=2^{-6}$ and $h_{c}=2^{-7}$. In this case, we have used an adaptive time step

$$
\Delta t=h_{c} \min \left(1, \frac{1}{\max \left\|\mathbf{u}_{s p}\right\|}\right)
$$

which always leads to an $\mathscr{F}_{6,8}(\cdot)$-compatible $\Delta t=h_{c}$ for $\ell_{c}^{\max }=6$ but not for $\ell_{c}^{\max }=7$. Qualitatively, the results in figs. 15 and 16 show that our hybrid solver converges to the baseline solutions at both levels of refinement. These plots thus suggest that our hybrid strategy yields trajectories that agree with the numerical patterns obtained with $\ell_{c}^{\max }=6$ and $\ell_{c}^{\max }=7$ for curvature-driven simulations. But, to understand better the machine learning effects on crystallization, further research is necessary to relax the velocity maximum-unit-norm restriction. Possible paths to address this problem involve increasing the variety of speed patterns in $\mathscr{D}$ or introducing velocity embeddings or encodings.

\section{Conclusions}

We have introduced a hybrid machine learning strategy to improve the accuracy of semi-Lagrangian schemes for relatively coarse adaptive Cartesian grids. Our approach comprises a multilayer perceptron $\mathscr{F}_{c, f}(\cdot)$ that processes velocity, level-set, and positional information to quantify the error in the interface trajectory. To develop this model, we have postulated the problem according to the image super-resolution construct [7]. The latter requires employing a high-resolution mesh to supply reference level-set values for neural optimization. Once trained, $\mathscr{F}_{c, f}(\cdot)$ operates alongside selective reinitialization and alternates with standard advection to reduce numerical diffusion. Unlike other machine learning solutions for passive scalar transport (e.g., [13, 64-66, 101]), our approach is localized in time and space. In particular, we have avoided complex architectures and costly evaluations by concentrating our effort only on vertices next to the interface. Also, our error-correcting neural network consumes data from the current time frame, thus relieving the system from buffering past states. Together, these features make our strategy attractive to parallel level-set frameworks (e.g., [57]), where communication among computing nodes can be expensive. 


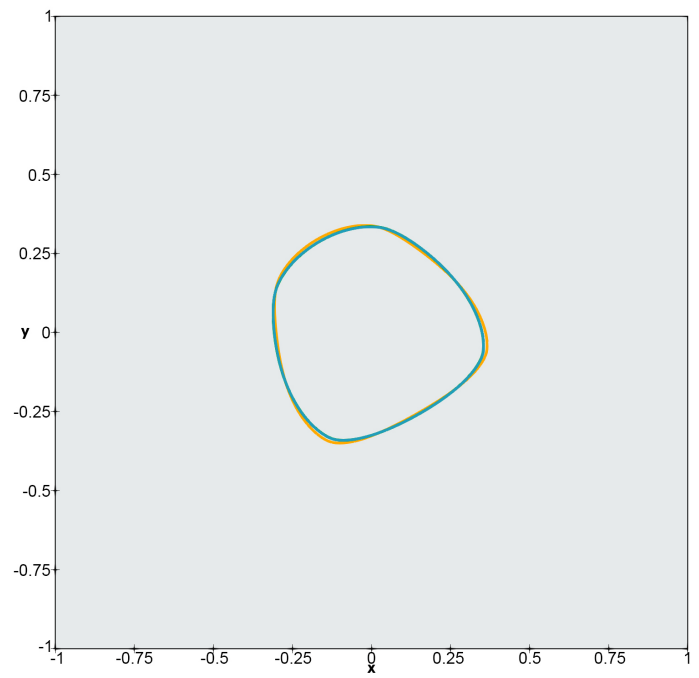

Figure 16: Comparison of the unstable-solidification zero level sets at time $t^{\text {end }}=3$. The numerical interfaces appear in green $\left(\ell_{c}^{\max }=6\right)$ and orange $\left(\ell_{c}^{\max }=7\right)$, while our hybrid approach solution is shown in blue for $h_{c}=2^{-6}$. The machine-learning-corrected and the standard zero-isocontours practically overlap. (Color online.)

We have also elaborated on the methodologies to assemble the learning data set and train the error-correcting neural network. Our main contribution is a novel machine-learning-augmented semi-Lagrangian algorithm that blends neural inference with numerically advected level-set values in an alternating fashion. To validate its correctness, we have examined the entire strategy with a few representative test cases. These included rotating and warping a disk, evaluating the effects of tangential shear flows, and two instances of the Stefan problem. Our results confirm that $\mathscr{F}_{c, f}(\cdot)$ can dampen numerical diffusion and improve mass loss in simple advection problems. More precisely, our strategy's accuracy is often as good as or better than the numerical scheme at twice the resolution while requiring only a fraction of the cost. Likewise, we have shown that our framework produces feasible solidification fronts for crystallization processes. However, bias artifacts and significant deterioration have occurred in lengthy or highly deforming simulations or in the presence of tangential shear flows. In addition, the stringent velocity maximum-unitnorm condition imposes severe limitations for FBPs involving rapid interface changes.

Unwelcome artifacts, unexpected results, and limitations in our case studies reveal several opportunities for future investigation. For example, we could enrich the learning data set by incorporating more than a single interface type (e.g., sine waves, as in [45]) and more velocity patterns, emphasizing under-resolved sampling. Other promising avenues for enhancing robustness involve binding physical constraints to $\mathscr{F}_{c, f}(\cdot)$ [24-26, 102], integrating velocity embeddings, adversarial training [8,13,101], enforcing consistency by detecting well-resolved stencils [29-31, 9597]), and even replacing the semi-Lagrangian formulation with a more accurate method. Similarly, we should consider temporal data only if it does not set back MLSemiLagrangian()'s performance. Then, we could evaluate our solver on more complex flows, such as the Kirchhoff elliptical vortex [103] and Chaplygin's vortical structures [104-106]. As a separate, challenging task, it also remains to migrate our strategy to higher resolutions and three-dimensional domains. In the meantime, we echo the arguments and motivation in [66] and hope our contributions open up further interdisciplinary research between machine learning and scientific computing.

\section{Acknowledgements}

Use was made of computational facilities purchased with funds from the National Science Foundation (CNS1725797) and administered by the Center for Scientific Computing (CSC). The CSC is supported by the California NanoSystems Institute and the Materials Research Science and Engineering Center (MRSEC; NSF DMR 1720256) at UC Santa Barbara. 


\section{References}

[1] C. C. Aggarwal. Neural Networks and Deep Learning - A Textbook. Springer, Cham, 2018.

[2] P. Mehta, M. Bukov, C.-H. Wang, A. G. R. Day, C. Richardson, C. K. Fisher, and D. J. Schwab. A high-bias, low-variance introduction to machine learning for physicists. Phys. Rep., 810:1-124, May 2019.

[3] A. Géron. Hands-on Machine Learning with Scikit-Learn, Keras E TensorFlow: Concepts, Tools, and Techniques to Build Intelligent Systems. O'Reilly, 2nd edition, November 2019.

[4] M. Turk and A. Pentland. Eigenfaces for recognition. J. Cogn. Neurosci., 3(1):71-86, January 1991.

[5] P. Vincent, H. Larochelle, I. Lajoie, Y. Bengio, and P.-A. Manzagol. Stacked denoising autoencoders: Learning useful representations in a deep network with a local denoising criterion. J. Mach. Learn. Res., 11:3371-3408, December 2010.

[6] A. Krizhevsky, I. Sutskever, and G. E. Hinton. Imagenet classification with deep convolutional neural networks. In NIPS Conference, pages 1097-1105, 2012.

[7] C. Dong, C. C. Loy, K. He, and X. Tang. Learning a deep convolutional network for image super-resolution. In D. Fleet, T. Pajdla, B. Schiele, and T. Tuytelaars, editors, Computer Vision - ECCV 2014, pages 184-199, Cham, 2014. Springer International Publishing.

[8] I. J. Goodfellow, J. Pouget-Abadie, M. Mirza, B. Xu, D. Warde-Farley, S. Ozair, A. Courville, and Y. Bengio. Generative adversarial nets. In Z. Ghahramani, M. Welling, C. Cortes, N. D. Lawrence, and K. Q. Weinberger, editors, NIPS'14: Proceedings of the 27th International Conference on Neural Information Processing Systems, volume 2, pages 2672-2680. Curran Associates, Inc., December 2014.

[9] O. Ronneberger, P. Fischer, and T. Brox. U-Net: Convolutional networks for biomedical image segmentation. In N. Navab, J. Hornegger, W. M. Wells, and A. F. Frangi, editors, Medical Image Computing and Computer-Assisted Intervention - MICCAI 2015, pages 234-241, Cham, 2015. Springer International Publishing.

[10] K. He, X. Zhang, S. Ren, and J. Sun. Deep residual learning for image recognition. In CVPR, pages 770-778, 2016.

[11] T. A. Ngo, Z. Lu, and G. Carneiro. Combining deep learning and level set for the automated segmentation of the left ventricle of the heart from cardiac cine magnetic resonance. Medical Image Analysis, 35:159-171, January 2017.

[12] T. H. N. Le, K. G. Quach, K. Luu, C. N. Duong, and M. Savvides. Reformulating level sets as deep recurrent neural network approach to semantic segmentation. IEEE Trans. on Image Process., 27(5):2393-2407, May 2018.

[13] Y. Xie, E. Franz, M. Chu, and N. Thuerey. tempoGAN: A temporally coherent, volumetric GAN for super-resolution fluid flow. ACM Trans. Graph., 37(4):1-15, July 2018.

[14] T. Mikolov, I. Sutskever, K. Chen, G. Corrado, and J. Dean. Distributed representations of words and phrases and their compositionality. In C. J. C. Burges, L. Bottou, M. Welling, Z. Ghahramani, and K. Q. Weinberger, editors, NeurIPS 26, pages 3111-3119. Curran Associates, Inc., 2013.

[15] P. Bojanowski, E. Grave, A. Joulin, and T. Mikolov. Enriching word vectors with subword information. In Trans. Assoc. Comput. Linguist., volume 5, pages 135-146, 2017.

[16] M. E. Peters, M. Neumann, M. Iyyer, M. Gardner, C. Clark, K. Lee, and L. Zettlemoyer. Deep contextualized word representations. In Proc. of NAACL, 2018.

[17] S. Arora, Y. Liang, and T. Ma. A simple but tough-to-beat baseline for sentence embeddings. In Proc. ICLR, April 2017.

[18] S. Hochreiter and J. Schmidhuber. Long short-term memory. Neural Comput., 9(8):1735-1780, November 1997.

[19] K. Cho, B. van Merriënboer, C. Gülçehre, D. Bahdanau, F. Bougares, H. Schwenk, and Y. Bengio. Learning phrase representations using RNN encoder-decoder for statistical machine translation. In Proc. EMNLP, 2014.

[20] A. Vaswani, N. Shazeer, N. Parmar, J. Uszkoreit, L. Jones, A. N. Gomez, Ł. Kaiser, and I. Polosukhin. Attention is all you need. In I. Guyon, U. V. Luxburg, S. Bengio, H. Wallach, R. Fergus, S. Vishwanathan, and R. Garnett, editors, NeurIPS 30, pages 5998-6008. Curran Associates, Inc., 2017.

[21] N. Yadav, A. Yadav, and M. Kumar. An Introduction to Neural Network Methods for Differential Equations. SpringerBriefs in Computational Intelligence. Springer Netherlands, 2015.

[22] I. E. Lagaris, A. Likas, and D. I. Fotiadis. Artificial neural networks for solving ordinary and partial differential equations. IEEE Trans. Neural Netw., 9(5):987-1000, 1998.

[23] I. E. Lagaris, A. C. Likas, and D. G. Papageorgiou. Neural-network methods for boundary value problems with irregular boundaries. IEEE Trans. Neural Netw., 11(5):1041-1049, September 2000.

[24] M. Raissi, P. Perdikaris, and G. E. Karniadakis. Physics informed deep learning (part I): Data-driven solution of nonlinear partial differential equations. https://arxiv org/abs/1711.10561, November 2017.

[25] M. Raissi. Deep hidden physics models: Deep learning of nonlinear partial differential equations. J. Mach. Learn. Res., 19(25):1-24, July 2018.

[26] M. Raissi, P. Perdikaris, and G. E. Karniadakis. Physics-informed neural networks: A deep learning framework for solving forward and inverse problems involving nonlinear partial differential equations. J. Comput. Phys., 378:686-707, February 2019.

[27] W.-F. Hu, T.-S. Lin, and M.-C. Lai. A discontinuity capturing shallow neural network for elliptic interface problems. https://arxiv . org/abs/2106.05587, June 2021.

[28] S. Pakravan, P. A. Mistani, M. A. Aragon-Calvo, and F. Gibou. Solving inverse-PDE problems with physics-aware neural networks. J. Comput. Phys., 440(4):110414, September 2021.

[29] D. Ray and J. S. Hesthaven. An artificial neural network as a troubled-cell indicator. J. Comput. Phys., 367:166-191, August 2018.

[30] N. R. Morgan, S. Tokareva, X. Liu, and A. Morgan. A machine learning approach for detecting shocks with high-order hydrodynamic methods. AIAA SciTech Forum, January 2020.

[31] A. B. Buhendwa, D. A. Bezgin, and N. A. Adams. Consistent and symmetry preserving data-driven interface reconstruction for the level-set method. J. Comput. Phys., 457:111049, May 2022. 
[32] A. Friedman. Variational Principles of Free-Boundary Problems. Dover Publications, 2010.

[33] C. W. Hirt and B. D. Nichols. Volume of fluid (VOF) method for the dynamics of free boundaries. J. Comput. Phys., 39(1):201-225, January 1981.

[34] A. Fasano and M. Primicerio, editors. Free Boundary Problems: Theory and Applications. Pitman, Boston, 1983.

[35] J. S. Langer. Models of Pattern Formation in First-Order Phase Transitions. Number 165-186. World Scientific, 1986.

[36] S. Osher and J. A. Sethian. Fronts propagating with curvature-dependent speed: Algorithms based on Hamilton-Jacobi formulations. $J$. Comput. Phys., 79(1):12-49, November 1988.

[37] B. Després and H. Jourdren. Machine learning design of volume of fluid schemes for compressible flows. J. Comput. Phys., 408(1):109275, May 2020.

[38] Y. Qi, J. Lu, R. Scardovelli, S. Zaleski, and G. Tryggvason. Computing curvature for volume of fluid methods using machine learning. J. Comput. Phys., 377:155-161, January 2019.

[39] H. V. Patel, A. Panda, J. A. M. Kuipers, and E. A. J. F. Peters. Computing interface curvature from volume fractions: A machine learning approach. Comput. E Fluids, 193:104263, October 2019.

[40] M. Ataei, M. Bussmann, V. Shaayegan, F. Costa, S. Han, and C. B. Park. NPLIC: A machine learning approach to piecewise linear interface construction. Comput. E Fluids, 223:104950, June 2021.

[41] D. L. Youngs. Time-dependent multi-material flow with large fluid distortion. Num. Method Fluid Dyn., 24:273-285, January 1982.

[42] S. Popinet. Numerical models of surface tension. Annu. Rev. Fluid Mech., 50(1):49-75, January 2018.

[43] M. Moog, R. Keck, and A. Zemitis. Some numerical aspects of the level set method. Math. Model. Anal., 3(1):140-151, 1998.

[44] M. Sussman and E. G. Puckett. A coupled level set and volume-of-fluid method for computing 3D and axisymmetric incompressible twophase flows. J. Comput. Phys., 162(2):301-337, August 2000.

[45] L. Á. Larios-Cárdenas and F. Gibou. A deep learning approach for the computation of curvature in the level-set method. SIAM J. Sci. Comput., 43(3):A1754-A1779, May 2021.

[46] L. Á. Larios-Cárdenas and F. Gibou. A hybrid inference system for improved curvature estimation in the level-set method using machine learning. J. Comput. Phys., 463:111291, August 2022.

[47] M. J. Berger and J. Oliger. Adaptive mesh refinement for hyperbolic partial differential equations. J. Comput. Phys., 53(3):484-512, March 1984.

[48] J. Strain. Tree methods for moving interfaces. J. Comput. Phys., 151(2):616-648, May 1999.

[49] C. Min and F. Gibou. A second order accurate projection method for the incompressible Navier-Stokes equations on non-graded adaptive grids. J. Comput. Phys., 219(2):912-929, December 2006.

[50] C. Min, F. Gibou, and H. D. Ceniceros. A supra-convergent finite difference scheme for the variable coefficient Poisson equation on nongraded grids. J. Comput. Phys., 218(1):123-140, October 2006.

[51] C. Min and F. Gibou. A second order accurate level set method on non-graded adaptive Cartesian grids. J. Comput. Phys., 225(1):300-321, July 2007.

[52] H. Chen, C. Min, and F. Gibou. A supra-convergent finite difference scheme for the Poisson and heat equations on irregular domains and non-graded adaptive Cartesian grids. J. Sci. Comput., 31(1-2):19-60, March 2007.

[53] C. Min and F. Gibou. Geometric integration over irregular domains with application to level-set methods. J. Comput. Phys., 226(2):14321443, October 2007.

[54] C. Min and F. Gibou. Robust second-order accurate discretizations of the multi-dimensional Heaviside and Dirac delta functions. J. Comput. Phys., 227(22):9686-9695, November 2008.

[55] H. Chen, C. Min, and F. Gibou. A numerical scheme for the Stefan problem on adaptive Cartesian grids with supralinear convergence rate. J. Comput. Phys., 228(16):5803-5818, September 2009.

[56] C. Burstedde, L. C. Wilcox, and O. Ghattas. p4est: Scalable algorithms for parallel adaptive mesh refinement on forests of octrees. SIAM J. Sci. Comput., 33(3):1103-1133, May 2011.

[57] M. Mirzadeh, A. Guittet, C. Burstedde, and F. Gibou. Parallel level-set methods on adaptive tree-based grids. J. Comput. Phys., 322:345-364, October 2016.

[58] D. Enright, R. Fedkiw, J. Ferziger, and I. Mitchell. A hybrid particle level set method for improved interface capturing. J. Comput. Phys., 183(1):83-116, November 2002.

[59] D. L. Sun and W. Q. Tao. A coupled volume-of-fluid and level set (VOSET) method for computing incompressible two-phase flows. Int. J. Heat Mass Transf., 53(4):645-655, January 2010.

[60] V. Mihalef, D. Metaxas, and M. Sussman. Textured liquids based on the marker level set. Comput. Graph. Forum, 26(3):457-466, September 2007.

[61] A. Salih and S. Ghosh Moulic. A mass conservation scheme for level set method applied to multiphase incompressible flows. Int. J. Comput. Meth. Eng. Sci. Mech., 14(4):271-289, May 2013.

[62] H.-Z. Yuan, C. Shu, Y. Wang, and S. Shu. A simple mass-conserved level set method for simulation of multiphase flows. Phys. Fluids, 30(4):040908, March 2018.

[63] Y. Bar-Sinai, S. Hoyer, J. Hickey, and M. P. Brenner. Learning data-driven discretizations for partial differential equations. PNAS, 116(31):15344-15349, July 2019.

[64] J. Zhuang, D. Kochkov, Y. Bar-Sinai, M. P. Brenner, and S. Hoyer. Learned discretizations for passive scalar advection in a two-dimensional turbulent flow. Phys. Rev. Fluids, 6(6):064605, June 2021. https://github. com/google-research/data-driven-advection.

[65] B. Liu, J. Tang, H. Huang, and X.-Y. Lu. Deep learning methods for super-resolution reconstruction of turbulent flows. Phys. Fluids, 32(2):025105, February 2020. 
[66] J. Pathak, M. Mustafa, K. Kashinath, E. Motheau, T. Kurth, and M. Day. Using machine learning to augment coarse-grid computational fluid dynamics simulations. https://arxiv. org/abs/2010.00072, October 2020.

[67] R. Courant, E. Isaacson, and M. Rees. On the solution of nonlinear hyperbolic differential equations by finite differences. Comm. Pure Appl. Math., 5:243-255, August 1952.

[68] A. Wiin-Nielsen. On the application of trajectory methods in numerical forecasting. Tellus, 11(2):180-196, 1959.

[69] S. J. Fletcher. Semi-Lagrangian Advection Methods and Their Applications in Geoscience. Elsevier, Amsterdam, Netherlands; Oxford, England; Cambridge, Massachusetts, 2020.

[70] M. Sussman, P. Smereka, and S. Osher. A level set approach for computing solutions to incompressible two-phase flow. J. Comput. Phys., 114(1):146-159, September 1994.

[71] C. Min. On reinitializing level set functions. J. Comput. Phys., 229(8):2764-2772, April 2010.

[72] S. Osher and R. Fedkiw. Level Set Methods and Dynamic Implicit Surfaces. Appl. Math. Sci. 153. Springer, Cham, 2002.

[73] J. A. Sethian. Level Set Methods and Fast Marching Methods. Cambridge Monogr. Appl. Comput. Math. Cambridge University Press, Cambridge, UK, 2nd edition, 1999.

[74] F. Gibou, R. Fedkiw, and S. Osher. A review of level-set methods and some recent applications. J. Comput. Phys., 353:82-109, January 2018.

[75] M. de Berg, M. van Kreveld, M. Overmars, and O. Schwarzkopf. Computational Geometry - Algorithms and Applications. Springer, Cham, 2nd edition, 2000.

[76] C. Min. Local level set method in high dimension and codimension. J. Comput. Phys., 200(1):368-382, October 2004.

[77] W. Gropp, E. Lusk, and A. Skjellum. Using MPI: Portable Parallel Programming with the Message-Passing Interface. MIT Press, Cambridge, MA, 3rd edition, 2014.

[78] D. Xiu and G. E. Karniadakis. A semi-Lagrangian high-order method for Navier-Stokes equations. J. Comput. Phys, 172(2):658-684, September 2001.

[79] C.-W. Shu and S. Osher. Efficient implementation of essentially non-oscillatory shock capturing schemes. J. Comput. Phys., 77(2):439-471, August 1988.

[80] D. S. Parker. Exploring the Matrix - Adventures in Modeling with Matlab. UCLA Course Reader Solutions, January 2016.

[81] S. Balay, S. Abhyankar, M. F. Adams, J. Brown, P. Brune, K. Buschelman, L. Dalcin, A. Dener, V. Eijkhout, W. D. Gropp, D. Karpeyev, D. Kaushik, M. G. Knepley, D. A. May, L. Curfman McInnes, R. Tran Mills, T. Munson, K. Rupp, P. Sanan, B. F. Smith, S. Zampini, H. Zhang, and H. Zhang. PETSc. https://www.mcs . anl.gov/petsc, September 2019. v3.12.0.

[82] T. Saad and J. C. Sutherland. Comment on "Diffusion by a random velocity field" [Phys. Fluids 13, 22 (1970)]. Phys. Fluids, 28(11):119101, December 2016.

[83] A. du Chéné, C. Min, and F. Gibou. Second-order accurate computation of curvatures in a level set framework using novel high-order reinitialization schemes. J. Sci. Comput., 35:114-131, June 2008.

[84] M. Abadi, A. Agarwal, P. Barham, E. Brevdo, Z. Chen, C. Citro, G. S. Corrado, A. Davis, J. Dean, M. Devin, S. Ghemawat, I. Goodfellow, A. Harp, G. Irving, M. Isard, R. Jozefowicz, Y. Jia, L. Kaiser, M. Kudlur, J. Levenberg, D. Mané, M. Schuster, R. Monga, S. Moore, D. Murray, C. Olah, J. Shlens, B. Steiner, I. Sutskever, K. Talwar, P. Tucker, V. Vanhoucke, V. Vasudevan, F. Viégas, O. Vinyals, P. Warden, M. Wattenberg, M. Wicke, Y. Yu, and X. Zheng. TensorFlow: Large-scale machine learning on heterogeneous systems. https://www . tensorflow.org, 2015.

[85] F. Chollet et al. Keras. https://keras.io, 2015.

[86] F. Pedregosa, G. Varoquaux, A. Gramfort, V. Michel, B. Thirion, O. Grisel, M. Blondel, P. Prettenhofer, R. Weiss, V. Dubourg, J. Vanderplas, A. Passos, D. Cournapeau, M. Brucher, M. Perrot, and E. Duchesnay. Scikit-learn: Machine learning in Python. J. Mach. Learn. Res., 12:2825-2830, 2011.

[87] W. McKinney. Data structures for statistical computing in Python. In S. van der Walt and J. Millman, editor, Proceedings of the 9th Python in Science Conference, pages 56-61, June 2010. https: //pandas . pydata.org.

[88] Y. A. LeCun, L. Bottou, G. B. Orr, and K.-R. Müller. Efficient BackProp, volume 7700 of Lecture Notes in Comput. Sci., pages $9-48$. Springer Berlin Heidelberg, Berlin, Heidelberg, 2012.

[89] I. Goodfellow, Y. Bengio, and A. Courville. Deep Learning. MIT Press, 2016. https : //www . deeplearningbook. org.

[90] D. P. Kingma and J. L. Ba. Adam: A method for stochastic optimization. ICLR, pages 1-13, May 2015.

[91] N. Lohmann. JSON for modern C++. https://github.com/nlohmann/json, August 2020. v3.9.1.

[92] T. Hermann. Frugally-deep. https://github.com/Dobiasd/frugally-deep, February 2021. v0.15.2.

[93] Z. Xianyi and M. Kroeker. OpenBLAS: An optimized BLAS library. https://github.com/xianyi/OpenBLAS, July 2021. v0.3.17.

[94] J. B. Bell, P. Colella, and H. M. Glaz. A second order projection method for the incompressible Navier-Stokes equations. J. Comput. Phys, 85(2):257-283, December 1989.

[95] P. Macklin and J. Lowengrub. An improved geometry-aware curvature discretization for level set methods: Application to tumor growth. $J$. Comput. Phys., 215(2):392-401, July 2006.

[96] K. Y. Lervåg. Calculation of interface curvature with the level-set method. https ://arxiv. org/abs/1407.7340, July 2014.

[97] Å. Ervik, K. Y. Lervåg, and S. T. Munkejord. A robust method for calculating interface curvature and normal vectors using an extracted local level set. J. Comput. Phys., 257, Part A:259-277, January 2014.

[98] V. Alexiades, A. D. Solomon, and D. G. Wilson. The formation of a solid nucleus in supercooled liquid, I. J. Non-Equilib. Thermodyn., 13:281-300, 1988.

[99] V. Alexiades and A. D. Solomon. Mathematical Modeling of Melting and Freezing Processes. Hemisphere, Washington, DC, 1st edition, 1993. 
[100] F. C. Frank. Radially symmetric phase growth controlled by diffusion. Proc. Royal Soc. A, 201(1067):586-599, May 1950.

[101] A. B. Farimani, J. Gomes, and V. S. Pande. Deep learning the physics transport phenomena. https://arxiv.org/abs/1709.02432, September 2017.

[102] T. Beucler, M. Pritchard, S. Rasp, J. Ott, P. Baldi, and P. Gentine. Enforcing analytic constraints in neural networks emulating physical systems. Phys. Rev. Lett., 126(9):098302, March 2021.

[103] G. Kirchhoff. Vorlesungen Über Mathematische Physik: Mechanik. Teubner, Leipzig, 1876.

[104] S. A. Chaplygin. On a pulsating cylindrical vortex. Trudy Otd. Fiz. Nauk Mosk. Obshch. Lyub. Estest., 10(1):13-22, 1899.

[105] S. A. Chaplygin. A case of vortex motion in a fluid. Trudy Otd. Fiz. Nauk Mosk. Obshch. Lyub. Estest., 11(2):11-14, 1903.

[106] V. V. Meleshko and G. J. F. van Heist. On Chaplygin's investigations of two-dimensional vortex structures in an inviscid fluid. J. Fluid Mech., 272:157-182, August 1994. 